\title{
Dynamics of a tagged particle in the asymmetric exclusion process with the step initial condition
}

\author{
T. Imamura * and T. Sasamoto ${ }^{\dagger}$ \\ * Institute of Industrial Science, University of Tokyo, \\ Komaba 4-6-1, Meguro-ku, Tokyo 153-8505, Japan \\ ${ }^{\dagger}$ Department of Mathematics and Informatics, \\ Chiba University, \\ Yayoi-cho 1-33, Inage-ku, Chiba 263-8522, Japan
}

\begin{abstract}
The one-dimensional totally asymmetric simple exclusion process (TASEP) is considered. We study the time evolution property of a tagged particle in the TASEP with the step initial condition. Calculated is the multi-time joint distribution function of its position. Using the relation of the dynamics of the TASEP to the Schur process, we show that the function is represented as the Fredholm determinant. We also study the scaling limit. The universality of the largest eigenvalue in the random matrix theory is realized in the limit. When the hopping rates of all particles are the same, it is found that the joint distribution function converges to that of the Airy process after the time at which the particle begins to move. On the other hand, when there are several particles with small hopping rate in front of a tagged particle, the limiting process changes at a certain time from the Airy process to the process of the largest eigenvalue in the Hermitian multi-matrix model with external sources.
\end{abstract}

[Keywords: asymmetric simple exclusion process; KPZ universality class; random matrices; Tracy-Widom distribution; Airy process]

*e-mail: timamura@iis.u-tokyo.ac.jp

${ }^{\dagger}$ e-mail: sasamoto@math.s.chiba-u.ac.jp 


\section{Introduction}

Dynamics of a nonequilibrium system is one of the most stimulating topics in statistical mechanics. The dynamical property is determined by the interplay among various elements such as interaction, initial and boundary conditions, and so on. But little is known about how it depends on these elements and realizes universality in a situation where the elements are intertwined. The aim of this article is to approach such questions by analyzing the dynamics of the one-dimensional asymmetric simple exclusion process (ASEP).

The ASEP is one of the most typical models of interacting particle processes where particles diffuse to a preferred direction with hard core repulsive interaction $[1,2,3,4,5]$. In spite of the simplicity of the model, it has been known that the ASEP shows various interesting phenomena caused by the collaboration between the diffusion and exclusion effect. Furthermore, in the one-dimensional case, it has an integrable mathematical structure which allows us to analyze some physical quantities exactly. For example, in the open boundary condition a steady state can be constructed using the matrix product method and $q$-orthogonal polynomials, and the boundary-induced phase transition can be discussed $[6,7,8]$.

On the other hand, the dynamical (non-stationary) properties of the one-dimensional ASEP have been also analyzed recently. Among various studies on the topic, we focus on the problem of the diffusion of a particular particle in the one-dimensional ASEP. (We call the particle a tagged particle.) This is a fundamental and elementary problem for the understanding of the dynamics of the ASEP. We are interested in how initial conditions and the exclusion effect through hard-core repulsive interaction affect the diffusion property and how it is different from the (normal) diffusion of the Brownian particle.

The scaling exponent of the diffusion provides insights into these problems. It was found that it depends on the initial conditions. In a steady state with a given density, the position fluctuation of a tagged particle grows as $t^{1 / 2}[9]$. The exponent $1 / 2$ is the same as that of the Brownian particle. On the other hand, for the fixed initial conditions where initially we create particles with a given density and fix the initial configuration, the exponent changes from $1 / 2$ to $1 / 3[10,11]$. Such a diffusion is called an anomalous diffusion. This exponent $1 / 3$ can be understood from the fact that the time evolution of the one-dimensional ASEP can be described by the one-dimensional Kardar-Parisi-Zhang universality class [12]. In the study of the current in the one-dimensional ASEP on a ring, the exponent $1 / 3$ was calculated by the Bethe ansatz technique $[13,14]$.

Note that the two cases above have a common property that the density is invariant under both time and space translation and a tagged particle moves with a fixed velocity on average. On the other hand, let us now consider another initial condition where all sites on the left of a certain bond are occupied while all right sites are empty. We call this initial condition a step initial condition. This is the typical initial condition where the density and the average velocity of a tagged particle change with time. Under the initial condition, we focus on the $M$ th particle from the right as a tagged particle and consider the time evolution of the particle when $M$ is large (but finite). In order to study this problem, we investigate the limiting behavior of the diffusion of the tagged particle as both $M$ and 
time go to infinity. In the totally asymmetric simple exclusion process (TASEP), where particles move only to the right, the average position of a tagged particle has been given in the scaling limit [15]. On the other hand, we are interested in the limiting process itself which the tagged particle obeys, as well as the average position and the scaling exponent.

Such an attempt as getting more sophisticated information than the scaling exponent has been recently discussed in the study on the current fluctuations in the TASEP. Not only the exponent but also the scaling function were obtained and it was revealed that the function is equivalent to the largest eigenvalue distribution in the random matrix theory with various universality classes. This development is based on the results on the longest increasing subsequence in a random permutation $[16,17,18,19,20]$.

Remarkable is that the scaling function detects a difference in initial conditions even if the scaling exponent does not. For instance, in the case of the step initial condition, the scaling function is equivalent to the GUE Tracy-Widom distribution [21], which is the largest eigenvalue distribution of the Gaussian unitary ensemble (GUE) [17, 22, 23]. In other initial conditions, on the other hand, these are described as the largest eigenvalue distributions with other universality classes in RMT such as Gaussian orthogonal ensemble (GOE) [24], two independent GOE's which is denoted by $\operatorname{GOE}^{2}[19,20]$, and so on [22, 25]. In addition, the equal-time multipoint distribution of the current fluctuations is obtained in the step and periodic initial conditions [26] by use of the Green function of the TASEP [27].

In this article, we calculate the multi-time distribution function of position fluctuations of a tagged particle in the TASEP with the step initial condition based on the techniques of the directed polymer problem in a 01 matrix $[28,29,30,31]$ and stochastic growth of Young diagram characterized by the Schur process [32, 33, 34, 35, 36, 37]. We express the function as the Fredholm determinant and discuss the scaling limit by the saddle point analysis. We remark that what will be considered in this paper is the pointwise convergence of the kernel. The convergence of the Fredholm determinant itself is certainly expected to hold from previous works on related problems [38, 39], but more elaborate asymptotic analysis is necessary for its proof. In the study on the ASEP, such correlations between different times have not been studied yet although the Green function [23, 27, 40] and the equal-time joint distributions of particle positions [39, 41] have been studied recently. In the one-time case, on the other hand, the position fluctuation is essentially the same as the current fluctuation which has already been studied in $[17,25]$. But it has not been stated as the position fluctuation.

Furthermore, we also consider the dependence of the distribution function on defect particles located in front of the tagged particle. Here defect particle means a particle with different hopping rate from other normal particles and we assume the number of them is finite. Such a situation as the TASEP with particle-dependent hopping rates has been recently discussed in $[42,43,44]$. We clarify that when their hopping rate is smaller than that of normal particles, the limiting behavior of the multi-time distribution changes due to their presence although their number is finite.

The paper is organized as follows. In the next section, we describe the definition of the model. The main result of this article is given in Section 3. In Section 4, we express the multi-time distribution of position fluctuations in the TASEP as Fredholm determinant. 
For this purpose, we first show that the directed polymer problem on a 01 matrix is related to the time evolution of a tagged particle in the TASEP with the step initial condition. Next, by applying the dual Robinson-Schensted-Knuth algorithm to the 01 matrix, we map the tagged particle problem in the TASEP to the stochastic growth of Young diagram described by the Schur process. In Section 5, we discuss the two types of scaling limit for the multi-time distribution function, the case where both time $t$ and the label of the tagged particle $M$ tend to infinity, and the one where $t$ goes to infinity with $M$ fixed. Some discussions and concluding remark are given in Section 6 and Section 7 respectively.

\section{Model}

In this section, we define the model and the quantity which we study in this article. Let us consider the one-dimensional infinite lattice and particles as illustrated in Fig. 1. Each site can be occupied by at most one particle. Suppose all particles are labeled an integer $i$ from the right. A particle moves stochastically obeying the following rules. During each time step between $t \in\{0,1, \cdots\}$ and $t+1$, the particle labeled $i$ hops to the right neighboring site with probability $1-q_{i}\left(0 \leq q_{i}<1\right)$ and stays at the same site with probability $q_{i}$ if the right neighboring site is empty. On the other hand, if the site is occupied by the particle labeled $i-1$, the $i$ th particle stays at the same site with probability 1 . This incorporates the exclusion effect which describes the hard core repulsive interaction. When hopping rates of all particles are the same, i.e., $q_{i}=q(\forall i)$, the system is the usual totally asymmetric simple exclusion process (TASEP) with parallel update. We allow the particle-dependent hopping rates and study the effects of defect particles.

In this article, we consider the step initial condition in which all sites on the left of a certain bond between two sites are occupied by particles and all sites on the right of the bond are empty. Fig. 1(b) illustrates the initial condition.

Under these settings, we label the particles at $1,2, \cdots$ from the right (see Fig. 1(b)) and consider the dynamics of the particle labeled $M$. (This particle which we focus on is called the tagged particle.) For this purpose, we set the position coordinate such that the tagged particle is at the origin at time 0 as depicted in Fig. 1(b). Let us define $L(t, M)$ as the position of the $M$ th particle at time $t$. In other words, $L(t, M)$ represents the distance travelled by the $M$ th particle from time 0 through $t$.

The quantity which we will investigate is the multi-time joint distribution function for $L(t, M)$ defined as

$$
\operatorname{Prob}\left(L\left(t_{1}, M\right) \geq \ell_{1}, L\left(t_{2}, M\right) \geq \ell_{2}, \cdots, L\left(t_{m}, M\right) \geq \ell_{m}\right) .
$$

In particular, we are interested in the asymptotic behavior of (2.1) as $t$ goes to infinity. In this article, two types of scaling limit will be discussed. The first one is the limit where both time $t$ and the label of the tagged particle $M$ go to infinity with the ratio $t / M$ fixed. In the second one, on the other hand, we take the $t \rightarrow \infty$ limit with $M$ fixed.

In order to see how the whole particles from the first to $M$ th travel, we performed the numerical simulation of which the result is shown in Fig. 2. We considered the time 
evolution of the particles from the first to 100th and assumed the hopping rate of all particles is 0.9 except the first, 25th, 50th and 75th particles whose hopping rate is 0.8 . Thus these four particles represent the defect particles. The typical example of the time evolution of the 100 particles from time 0 through 3000 is illustrated in Fig. 2(a). In this figure, the vertical axis represents the position coordinate introduced in Fig. 1(b), whereas the horizontal axis is time axis, and each particle is expressed as + . From the macroscopic point of view such as Fig. 2(a), we only find that they travel in a group. However, if we zoom the configuration of the particles in a certain time zone, we can see the microscopic pattern of the configuration. Here we show two characteristic configurations which are depicted in Fig. 2(b) and 2(c). Fig. 2(b) (resp.(c)) represents the configuration of the particles when time is around 200 (resp. 3000). When the time zone which we focus on is early enough as in Fig. 2(b), the particles move forming only one group and the four defect particles are included in the group. On the other hand, when time has passed sufficiently as in Fig. 2(c), they form the four groups automatically and the top of each group is the defect particle. The similar platoon structure was also discussed in the study of the TASEP with disordered hopping rates $[45,46,47]$. In fact, as will be explained in the next section, the position fluctuation of a tagged particle also changes with time corresponding to the change of the configuration.

\section{Main results}

\subsection{Multi-time distributions}

Our whole discussions in this article are based on a fact that the tagged particle problem for the step initial condition has a nice combinatorial structure which admits us to obtain a closed expression for the multi-time joint distribution in the form of the Fredholm determinant. The results are summarized in the following theorem. The proof will be presented in Section 4.

Note that due to the rule of the TASEP, the particle labeled $M$ cannot move when $t<M$. Thus, throughout the article, we consider the multi-time function (2.1) under the condition

$$
t_{i} \geq M
$$

\section{Theorem 1}

When (3.1) holds,

$$
\operatorname{Prob}\left(L\left(t_{1}, M\right) \geq \ell_{1}, L\left(t_{2}, M\right) \geq \ell_{2}, \cdots, L\left(t_{m}, M\right) \geq \ell_{m}\right)=\operatorname{det}(1+K g) .
$$


Here $\operatorname{det}(1+K g)$ is the Fredholm determinant defined as

$$
\begin{aligned}
& \operatorname{det}(1+K g) \\
& =\sum_{k=0}^{\infty} \frac{1}{k !} \sum_{n_{1}=1}^{m} \sum_{x_{1}=-\infty}^{\infty} \cdots \sum_{n_{k}=1}^{m} \sum_{x_{k}=-\infty}^{\infty} g\left(t_{n_{1}} ; x_{1}\right) \cdots g\left(t_{n_{k}} ; x_{k}\right) \operatorname{det}\left(K\left(t_{n_{l}}, x_{l} ; t_{n_{l^{\prime}}}, x_{l^{\prime}}\right)\right)_{l, l^{\prime}=1}^{k},
\end{aligned}
$$

where

$$
\begin{aligned}
g\left(t_{n} ; x\right) & =-\chi_{\left(t_{n}-M+1-\ell_{n}, \infty\right)}(x), \quad(n=1,2, \ldots, m), \\
\chi_{(a, b)}(x) & = \begin{cases}1, & \text { if } a<x<b, \\
0, & \text { otherwise },\end{cases}
\end{aligned}
$$

and the kernel $K\left(t_{1}, x_{1} ; t_{2}, x_{2}\right)$ is given by

$$
\begin{aligned}
& K\left(t_{1}, x_{1} ; t_{2}, x_{2}\right)=\tilde{K}\left(t_{1}, x_{1} ; t_{2}, x_{2}\right)-\phi_{t_{1}, t_{2}}\left(x_{1}, x_{2}\right), \\
& \tilde{K}\left(t_{1}, x_{1} ; t_{2}, x_{2}\right) \\
& =\frac{1}{(2 \pi i)^{2}} \int_{C_{R_{1}}} \frac{d z_{1}}{z_{1}} \int_{C_{R_{2}}} \frac{d z_{2}}{z_{2}} \frac{z_{1}}{z_{1}-z_{2}} \frac{\left(1+1 / z_{2}\right)^{t_{2}-M+1}}{\left(1+1 / z_{1}\right)^{t_{1}-M+1}} \prod_{i=1}^{M} \frac{1-q_{i}-q_{i} z_{2}}{1-q_{i}-q_{i} z_{1}} \frac{z_{2}^{x_{2}}}{z_{1}^{x_{1}}}, \\
& \phi_{t_{1}, t_{2}}\left(x_{1}, x_{2}\right)= \begin{cases}\frac{1}{2 \pi i} \int_{C_{1}} \frac{d z}{z}\left(1+\frac{1}{z}\right)^{t_{2}-t_{1}} z^{x_{2}-x_{1}}, & t_{1}<t_{2}, \\
0, & t_{1} \geq t_{2} .\end{cases}
\end{aligned}
$$

Here $C_{R}$ in (3.6) and (3.7) denotes a contour enclosing the origin anticlockwise with radius $R$ and $R_{i}(i=1,2)$ in (3.6) satisfy the conditions $R_{2}<R_{1}$ and $1<R_{1}<\left(1-q_{i}\right) / q_{i}$.

\subsection{Scaling limit $1(M \rightarrow \infty)$}

Using the results in Theorem 1, one can study the asymptotics of the joint distribution. In this subsection, we consider the scaling limit such that both $t$ and $M$ go to infinity with their ratio

$$
u=t / M
$$

fixed. $u$ represents the scaled time. Notice that we also take the limit of the label of the tagged particle $M$. This may sound strange if the particle we are focusing on is varied as $M$ goes to infinity. Rather we think that the results below give the asymptotic behaviors of the path statistics of the tagged particle for large but finite $M$ which is fixed. Alternatively one might be able to interpret them as giving the asymptotic behaviors of the path statistics of the tagged particle near a large but finite $t$.

We also discuss the effect of defect particles. In particular, we consider the situation where there are finite $n$ defect particles with stay rates $\bar{q}_{i}(i=1, \cdots, n)$ in front of the 
tagged particle. For this purpose, we set the stay rate $q_{i}$ of $i$ th particle as follows. For some set $\left\{a_{i}\right\}_{i=1, \cdots, n} \subset\{1,2, \cdots M\}$ with $n$ fixed, we assume

$$
q_{i}= \begin{cases}\bar{q}_{j}, & \text { if } i=a_{j} \\ q, & \text { otherwise }\end{cases}
$$

Note that the particle with the rate $\bar{q}_{j}$ represents the defect particle whose label is $a_{j}$ whereas the remaining particles with the rate $q$ are the normal particles.

\subsubsection{Average position}

The average position of the tagged particle divided by $M$ has a deterministic limit as $M \rightarrow \infty$. Let us call it $A(u)$,

$$
A(u)=\lim _{M \rightarrow \infty} \frac{L(t=u M, M)}{M} .
$$

We also define $\bar{q}=\max \left\{\bar{q}_{i}\right\}$. (Note that $\bar{q}$ is the stay rate of the slowest defect particle.) If $\bar{q}>q$, which is the case where the slowest defect particle is slower than the normal particles, we obtain

$$
A(u)= \begin{cases}0, & \text { if } u \leq \frac{1}{1-q} \\ A_{2}(u), & \text { if } \frac{1}{1-q} \leq u \leq u_{c} \\ A_{G}(u), & \text { if } u_{c} \leq u\end{cases}
$$

where $u_{c}=\left(\bar{q}^{2}-2 q \bar{q}+q\right) /(\bar{q}-q)^{2}$ and

$$
\begin{aligned}
& A_{2}(u)=(1-q) u-(1-2 q)-2 \sqrt{q(1-q)(u-1)}, \\
& A_{G}(u)=(1-\bar{q}) u-\frac{(1-\bar{q}) \bar{q}}{\bar{q}-q}
\end{aligned}
$$

$A_{2}(u)$ can be understood from Theorem 1.1. in [17]. If $\bar{q} \leq q$, the average position is represented as only the first and second cases in (3.11). $\left(u_{c}\right.$ and $A_{G}(u)$ do not appear in the case.) Note that $A_{2}(u)$ does not depend on the stay rate $\bar{q}$ of the slowest defect particle whereas $A_{G}(u)$ does. $A(u)$ is illustrated in Fig. 3.

Next we consider the position fluctuations of the tagged particle around the average position. There are four typical regions shown in Fig. 3. In each region, we can obtain the result on the position fluctuations by focusing on a point within the region and taking a proper scaling around the point. Note that in Fig. 3, the regions 2 and 4 spread in the form of a line whereas the regions 1 and 3 are point-like. Thus, in the region 1(resp. 3 ), we always consider the fluctuation property around the point $1 /(1-q)$ (resp. $u_{c}=$ $\left.\left(\bar{q}^{2}-2 q \bar{q}+q\right) /(\bar{q}-q)^{2}\right)$. 


\subsubsection{Region $1(u=1 /(1-q))$}

This is the region where the tagged particle just starts to move. This corresponds to $u=1 /(1-q)$. We set

$$
t_{i}=\frac{M}{1-q}+D_{1} M^{\frac{1}{2}} \tau_{i}
$$

where $D_{1}=\sqrt{q} /(1-q)$. Note that the time $t_{i}(3.14)$ is approximately equal to the time on which some leftmost holes arrive at the site occupied by the tagged particle. One easily finds that the arrival time can be scaled as in (3.14) which is the same scaling as the central limit theorem.

Applying the scaling to Theorem 1, we have the theorem as follows. The proof will be given in Section 5.1.

\section{Theorem 2-1}

$$
\lim _{M \rightarrow \infty} \operatorname{Prob}\left(L\left(t_{1}, M\right) \geq \ell_{1}, L\left(t_{2}, M\right) \geq \ell_{2}, \cdots, L\left(t_{m}, M\right) \geq \ell_{m}\right)=\operatorname{det}\left(1+\mathcal{K}_{1} g_{1}\right) .
$$

Here the Fredholm determinant in the right hand side is defined in (3.3) where $g_{1}\left(\tau_{i}, x\right)=$ $-\chi_{\left(-\infty, \ell_{i}\right)}(x) \cdot \chi_{(a, b)}(x)$ is defined in (3.4). The kernel is given by

$$
\mathcal{K}_{1}\left(\tau_{1}, x_{1} ; \tau_{2}, x_{2}\right)= \begin{cases}\sum_{m=0}^{\infty} \psi_{1}\left(x_{1}-m, \tau_{1}\right) \psi_{2}\left(x_{2}-m, \tau_{2}\right), & \tau_{1} \geq \tau_{2}, \\ -\sum_{m=0}^{\infty} \psi_{1}\left(x_{1}+m+1, \tau_{1}\right) \psi_{2}\left(x_{2}+m+1, \tau_{2}\right), & \tau_{1}<\tau_{2} .\end{cases}
$$

Here $\psi_{1}\left(x_{1}, \tau_{1}\right)$ can be represented by use of the parabolic cylinder function $D_{n}(x)$ in [48] as

$$
\psi_{1}\left(x_{1}, \tau_{1}\right)=\frac{1}{2 \pi i} \int_{-i \infty+\epsilon}^{i \infty+\epsilon} d z e^{\frac{z^{2}}{2}-\tau_{1} z} z^{x_{1}-1}=\frac{e^{-\frac{x_{1}^{2}}{4}}}{\sqrt{2 \pi}} D_{x_{1}-1}(\tau),
$$

where $\epsilon$ is taken to be positive, and

$$
\psi_{2}\left(x_{2}, \tau_{2}\right)=\frac{1}{2 \pi i} \int_{C_{1}} \frac{d w}{w^{x_{2}+1}} e^{-\frac{w^{2}}{2}+\tau_{2} w}= \begin{cases}\frac{2^{\frac{-x_{2}}{2}}}{x_{2} !} H_{x_{2}}\left(\frac{\tau}{\sqrt{2}}\right), & x_{2} \geq 0 \\ 0, & x_{2}<0\end{cases}
$$

where $C_{1}$ in (3.18) represents the contour enclosing the origin anticlockwise with radius 1 , and $H_{n}(x)$ is the Hermite polynomial with degree $n$ [48].

The special case where $\tau_{1}=\cdots=\tau_{m}=0$ has appeared in the distribution of the height fluctuation in the "critical regime" of the oriented digital boiling model [30]. Recently the

distribution in one time case $(m=1)$ has been also given in [49]. Our formula above gives a generalization to the multi-time version.

In particular, the limiting distribution in the case $m=1$ can be described as

$$
\lim _{M \rightarrow \infty} \operatorname{Prob}(L(t, M) \geq \ell)=\sum_{k=0}^{\ell} \frac{(-1)^{k}}{k !} \sum_{x_{1}=0}^{\ell-1} \cdots \sum_{x_{k}=0}^{\ell-1} \operatorname{det}\left(\mathcal{K}_{1}\left(\tau, x_{l} ; \tau, x_{l^{\prime}}\right)\right)_{l, l^{\prime}=1}^{k},
$$


where

$$
\mathcal{K}_{1}\left(\tau, x_{1} ; \tau, x_{2}\right)=\sum_{m=0}^{x_{2}} \psi_{1}\left(x_{1}-m, \tau\right) \psi_{2}\left(x_{2}-m, \tau\right) .
$$

Note that in (3.19) the summation on $k$ where $k \geq \ell+1$ can be omitted. Thus in this case, we can obtain exactly the limiting probability by calculating the determinants with finite rank. Another expression of the kernel (3.20) has recently appeared as the "discrete Hermite kernel" in [49]. In [30], the specific values of the probability are given in the case $\tau=0$.

\subsubsection{Region $2\left(1 /(1-q)<u<u_{c}\right)$}

This is the region where the effect of the defect particles does not affect the time evolution of the tagged particle and the dynamics of the ordinary TASEP is dominant. The typical picture of the time evolution of the whole particles from 1 st to $M$ th in this region is illustrated in Fig. 2(b). In this figure, we are interested in the position fluctuations of the bottommost particle.

Let us scale as

$$
\begin{aligned}
& t_{j}=u M+C(u) M^{\frac{2}{3}} \tau_{j}, \\
& \ell_{j}=A_{2}\left(u_{j}\right) M-D(u) M^{\frac{1}{3}} s_{i},
\end{aligned}
$$

where $1 /(1-q)<u<u_{c}$ and $u_{j}=t_{j} / M . A_{2}(u)$ is defined in (3.12) and

$$
\begin{aligned}
& C(u)=2(u-1)^{\frac{5}{6}}\left(1+\sqrt{\frac{1-q}{q(u-1)}}\right)^{\frac{1}{3}}\left(\sqrt{u-1}-\sqrt{\frac{q}{1-q}}\right)^{\frac{1}{3}}, \\
& D(u)=(u-1)^{\frac{1}{6}} q^{\frac{1}{2}}(1-q)^{\frac{1}{2}}\left(1+\sqrt{\frac{1-q}{q(u-1)}}\right)^{\frac{2}{3}}\left(\sqrt{u-1}-\sqrt{\frac{q}{1-q}}\right)^{\frac{2}{3}} .
\end{aligned}
$$

Note that the scaling exponent $1 / 3$ in (3.22) also appears in an anomalous diffusion of the tagged particle $[10,11]$. The scaling exponents $2 / 3$ and $1 / 3$ in $(3.21)$ and $(3.22)$ are characteristic of the one-dimensional KPZ universality class. They are expected to be universal for all models belonging to the KPZ universality class.

Under the scaling defined above, we get the following theorem for the scaling function. This function is also universal but may depend on various elements such as initial conditions and the effect of the defect particles. For example, as we will show in Theorem 2-3, the scaling function in the region 3 is different from that in region 2 although we take the same scaling (3.21) and (3.22) in both regions. Thus, the function provides more detailed viewpoint in the KPZ universality than the scaling exponents. The proof of this theorem will be given in Section 5.2.

\section{Theorem 2-2}

$$
\lim _{M \rightarrow \infty} \operatorname{Prob}\left(L\left(t_{1}, M\right) \geq \ell_{1}, L\left(t_{2}, M\right) \geq \ell_{2}, \cdots, L\left(t_{m}, M\right) \geq \ell_{m}\right)=\operatorname{det}\left(1+\mathcal{K}_{2} \mathcal{G}\right) .
$$


Here the right hand side is the Fredholm determinant defined as

$$
\begin{aligned}
& \operatorname{det}\left(1+\mathcal{K}_{2} \mathcal{G}\right) \\
& =\sum_{k=0}^{\infty} \frac{1}{k !} \sum_{n_{1}=1}^{m} \int_{-\infty}^{\infty} d \xi_{1} \cdots \sum_{n_{k}=1}^{m} \int_{-\infty}^{\infty} d \xi_{k} \mathcal{G}\left(\tau_{n_{1}}, \xi_{1}\right) \cdots \mathcal{G}\left(\tau_{n_{k}}, \xi_{k}\right) \operatorname{det}\left(\mathcal{K}_{2}\left(\tau_{n_{l}}, \xi_{l} ; \tau_{n_{l^{\prime}}}, \xi_{l^{\prime}}\right)\right)_{l, l^{\prime}=1}^{k}
\end{aligned}
$$

where $\mathcal{G}\left(\tau_{j}, \xi\right)(j=1, \cdots, m)$ are defined in terms of $\chi_{(a, b)}(x)(3.4)$, as

$$
\mathcal{G}\left(\tau_{j}, \xi\right)=-\chi_{\left(s_{j}, \infty\right)}(\xi)(j=1, \cdots, m),
$$

and the kernel $\mathcal{K}_{2}\left(\tau_{1}, \xi_{1} ; \tau_{2}, \xi_{2}\right)$ is given by

$$
\mathcal{K}_{2}\left(\tau_{1}, \xi_{1} ; \tau_{2}, \xi_{2}\right)= \begin{cases}\int_{0}^{\infty} d \lambda e^{-\lambda\left(\tau_{1}-\tau_{2}\right)} \operatorname{Ai}\left(\xi_{1}+\lambda\right) \operatorname{Ai}\left(\xi_{2}+\lambda\right), & \tau_{1} \geq \tau_{2}, \\ -\int_{-\infty}^{0} d \lambda e^{-\lambda\left(\tau_{1}-\tau_{2}\right)} \operatorname{Ai}\left(\xi_{1}+\lambda\right) \operatorname{Ai}\left(\xi_{2}+\lambda\right), & \tau_{1}<\tau_{2}\end{cases}
$$

The kernel $\mathcal{K}_{2}$ is called the extended Airy kernel $[50,51]$. The process characterized by the Fredholm determinant with this kernel is called the Airy process [38, 52]. This process appears as the limiting process of the largest eigenvalue in Dyson's Brownian motion model [53] of the unitary class. The model is described as $N \times N$ Hermitian matrix where each independent element of $H$ obeys the Ornstein-Uhlenbeck process. The transition probability density $\mathrm{P}\left(H_{i} ; H_{j} ; \tau\right)$ from matrix $H_{i}$ to $H_{j}$ during $\tau$ is given by

$$
\mathrm{P}\left(H_{i} ; H_{j} ; \tau\right)=Z_{\tau} \exp \left(\frac{-\operatorname{tr}\left\{H_{j}-e^{-\tau} H_{i}\right\}^{2}}{1-e^{-2 \tau}}\right)
$$

where $Z_{\tau}$ is the normalization constant. If we choose the initial matrix $H_{0}$ to be GUE random matrix, the joint density function of the probability that matrix $H_{j}$ is at time $t_{j}$ is represented as the Hermitian multi-matrix model,

$$
\begin{aligned}
& \mathrm{P}\left(H_{1}, t_{1} ; \cdots ; H_{m}, t_{m}\right) \\
& =Z \prod_{j=1}^{m} \exp \left(\frac{-\operatorname{tr}\left\{H_{j}-e^{t_{j-1}-t_{j}} H_{j-1}\right\}^{2}}{1-e^{2\left(t_{j-1}-t_{j}\right)}}\right) \exp \left(-\operatorname{tr} H_{0}^{2}\right)
\end{aligned}
$$

where $Z$ is the normalization constant. Let $l^{(i)}$ be the largest eigenvalue of $H_{i}$ and we consider the quantity,

$$
\operatorname{Prob}\left(l^{(1)} \leq a_{1}, \cdots, l^{(m)} \leq a_{m}\right)
$$

When we set

$$
t_{i}=\frac{\tau_{i}}{N^{\frac{1}{3}}}, a_{i}=\sqrt{2 N}+\frac{s_{i}}{\sqrt{2} N^{\frac{1}{6}}},
$$

the limiting distribution (3.31) is described as

$$
\lim _{N \rightarrow \infty} \operatorname{Prob}\left(l^{(1)} \leq a_{1}, \cdots, l^{(m)} \leq a_{m}\right)=\operatorname{det}\left(1+\mathcal{K}_{2} \mathcal{G}\right) .
$$


In the case $m=1$, the distribution is the GUE Tracy-Widom distribution [21].

Hence our Theorem 2-2 says that in the appropriate scaling limit the dynamics of the tagged particle in this region is equivalent to the dynamics of the largest eigenvalue of Dyson's Brownian motion of the unitary type. In the context of the one dimensional KPZ universality class, the Airy process has already appeared in the study of the polynuclear growth (PNG) model. In [38, 52], the process has first appeared as the equal-time multipoint height fluctuation. Recently in [34], it has been shown that the process also describes the correlations among any "space-like" points in the space-time plane. Our result is closely related to the latter situation.

\subsubsection{Region $3\left(u=u_{c}\right)$}

This region is the border between the region 2 where the normal particles in the TASEP are dominant, and the region 4 where only the finite number of defect particles are dominant.

First we consider the case where the stay rates $\bar{q}_{j}(j=1,2, \cdots, n)$ of the defect particles are distinct. Taking the same scaling as (3.21)-(3.24) with $u=u_{c}$, we obtain the following theorem.

\section{Theorem 2-3}

$$
\lim _{M \rightarrow \infty} \operatorname{Prob}\left(L\left(t_{1}, M\right) \geq \ell_{1}, L\left(t_{2}, M\right) \geq \ell_{2}, \cdots, L\left(t_{m}, M\right) \geq \ell_{m}\right)=\operatorname{det}\left(1+\mathcal{K}_{3} \mathcal{G}\right)
$$

where the Fredholm determinant and $\mathcal{G}\left(\tau_{j}, \xi\right)$ in the right hand side are defined in (3.26) and (3.27) respectively. The kernel is given by

$$
\begin{aligned}
& \mathcal{K}_{3}\left(\tau_{1}, \xi_{1} ; \tau_{2}, \xi_{2}\right) \\
= & \begin{cases}\mathcal{K}_{2}\left(\tau_{1}, \xi_{1} ; \tau_{2}, \xi_{2}\right)+\operatorname{Ai}\left(\xi_{2}\right) \int_{0}^{\infty} d \lambda e^{\tau_{1} \lambda} \operatorname{Ai}\left(\xi_{1}-\lambda\right), & \tau_{1}<0, \\
\mathcal{K}_{2}\left(\tau_{1}, \xi_{1} ; \tau_{2}, \xi_{2}\right)-\operatorname{Ai}\left(\xi_{2}\right) \int_{0}^{\infty} d \lambda e^{-\tau_{1} \lambda} \operatorname{Ai}\left(\xi_{1}+\lambda\right)+\operatorname{Ai}\left(\xi_{2}\right) e^{-\frac{\tau_{1}^{3}}{3}+\xi_{1} \tau_{2}}, & \tau_{1}>0,\end{cases}
\end{aligned}
$$

where $\mathcal{K}_{2}$ is the extended Airy kernel (3.28).

This kernel has appeared in $[54,55]$ in the study of the height fluctuation property of the PNG model with external sources. When $\tau_{1}=\cdots=\tau_{m}=0$, it has been known that the Fredholm determinant of this kernel is described as the distribution of the larger of the largest eigenvalues in two independent GOEs, which is denoted as $\mathrm{GOE}^{2}[56,57]$. On the other hand, when $\tau_{1}=\cdots=\tau_{m}=-\infty$, the Fredholm determinant becomes the GUE Tracy-Widom distribution. Thus (3.34) describes the transition of the largest eigenvalue distribution between GUE and GOE ${ }^{2}$.

Next we consider the situation where the hopping rates $\bar{q}_{j}(j=1,2, \cdots, n)$ are more or less the same. In addition to the scaling (3.21)-(3.24), we set the hopping rate of the defect particles as

$$
\bar{q}_{i}=\bar{q}-\bar{q}(1-\bar{q}) \frac{\eta_{i}}{D(u) M^{\frac{1}{3}}}
$$


where $D(u)$ is defined in (3.24). Note that the parameters $\eta_{i}(\geq 0)(i=1, \cdots, n)$ characterize the inhomogeneity of the hopping rates and the case $\eta_{1}=\eta_{2}=\cdots=\eta_{n}=0$ corresponds to the situation where the hopping rates degenerate completely.

Under these settings, we obtain the following theorem.

\section{Theorem 2-3'}

$$
\lim _{M \rightarrow \infty} \operatorname{Prob}\left(L\left(t_{1}, M\right) \geq \ell_{1}, L\left(t_{2}, M\right) \geq \ell_{2}, \cdots, L\left(t_{m}, M\right) \geq \ell_{m}\right)=\operatorname{det}\left(1+\mathcal{K}_{3}^{\prime} \mathcal{G}\right),
$$

where the Fredholm determinant and $\mathcal{G}\left(\tau_{j}, \xi\right)$ in the right hand side are defined in (3.26) and (3.27) respectively and the kernel is given by

$$
\begin{aligned}
\mathcal{K}_{3}^{\prime}\left(\tau_{1}, \xi_{1} ; \tau_{2}, \xi_{2}\right)= & \mathcal{K}_{2}\left(\tau_{1}, \xi_{1} ; \tau_{2}, \xi_{2}\right)+\sum_{j=1}^{n} \frac{1}{2 \pi} \int_{\Gamma_{3}} d w_{1} \exp \left(i \xi_{1} w_{1}+\frac{i w_{1}^{3}}{3}\right) \prod_{k=1}^{j} \frac{1}{\eta_{k}-\tau_{1}+i w_{1}} \\
& \times \frac{1}{2 \pi} \int_{-\infty}^{\infty} d w_{2} \exp \left(i \xi_{2} w_{2}+\frac{i w_{2}^{3}}{3}\right) \prod_{k=1}^{j-1}\left(\eta_{k}-\tau_{2}+i w_{2}\right)
\end{aligned}
$$

where the contour $\Gamma_{3}$ runs from $-\infty$ to $\infty$ passing the down side of the points $i\left(\eta_{k}-\tau_{1}\right)(k=$ $1, \cdots, n)$.

In the case $m=1$, this Fredholm determinant has appeared in [42, 43, 58]. Note that if we set $\eta_{j}=0$ for some $j$ and $\eta_{k}=\infty$ for $k \neq j$, we can realize the situation where the maximum of $q_{i}(i=1,2, \cdots, n)$ is unique such as the former case. Indeed in the setting above, the kernel $\mathcal{K}_{3}^{\prime}$ (3.38) is reduced to $\mathcal{K}_{3}$ (3.35). In that sense, the theorem is the generalization of Theorem 2-3. Later we will give only the proof of the theorem in Section 5.2.

The Fredholm determinant (3.37) also describes the limiting largest eigenvalue distribution in the following multi-matrix model. Let $\left\{H_{i}\right\}_{i=1, \cdots, m}$ be the $N \times N$ Hermitian matrices. Analogous to (3.30), the joint density function of the model is defined as

$$
\begin{aligned}
& \mathrm{P}\left(H_{1}, t_{1} ; \cdots ; H_{m}, t_{m}\right) \\
& =Z_{V} \exp \left(-\operatorname{tr} H_{0}^{2}\right) \prod_{j=1}^{m} \exp \left(\frac{-\operatorname{tr}\left\{H_{j}-e^{t_{j-1}-t_{j}} H_{j-1}\right\}^{2}}{1-e^{2\left(t_{j-1}-t_{j}\right)}}\right) \exp \left(\operatorname{tr} V H_{m}\right) .
\end{aligned}
$$

Here $Z_{V}$ is the normalization constant and $t_{i}(i=0, \cdots, m)$ are parameters of the model such that $t_{i-1}-t_{i}$ gauges the strength of the connection between matrix $H_{i-1}$ and $H_{i}$. $V=\operatorname{diag}\left(v_{1}, v_{2}, \cdots, v_{n}, 0,0, \cdots \cdots\right)$ represents the external source with rank $n$ of the model. Under the scalings (3.32) and

$$
v_{i}=\sqrt{2 N}\left(1-\frac{\eta_{i}}{N^{\frac{1}{3}}}\right),
$$

the limiting joint distribution of the largest eigenvalues $l^{(i)}$ of $H_{i}(i=1, \cdots, m)$ is described as the Fredholm determinant (3.37),

$$
\lim _{N \rightarrow \infty} \operatorname{Prob}\left(l^{(1)} \leq a_{1}, \cdots, l^{(m)} \leq a_{m}\right)=\operatorname{det}\left(1+\mathcal{K}_{3}^{\prime} \mathcal{G}\right) .
$$


In the case $n=1,(3.41)$ was shown in [55]. We can also show this equation for the general $n$ case in a similar manner.

\subsubsection{Region $4\left(u_{c}<u\right)$}

This is the region where the effect of the defect particles has become dominant for the dynamics of the tagged particle.

As in the region 3 , we first consider the case $\bar{q}_{1} \neq \bar{q}_{2} \neq \cdots \neq \bar{q}_{n}$. In this case, one can expect that the dynamics is effectively the same as that of the slowest defect particle with stay rate $\bar{q}=\max \left(\bar{q}_{j}\right)$. To see nontrivial correlations, times $t_{j}(j=1, \cdots, m)$ should be macroscopically separated. We set

$$
\ell_{j}=A_{G}\left(u_{j}\right) M-D_{G}\left(u_{j}\right) M^{\frac{1}{2}} s_{j}
$$

where $u_{j}=t_{j} / M, A_{G}\left(u_{j}\right)$ is given in (3.13), and

$$
D_{G}\left(u_{j}\right)=\frac{1-\bar{q}}{\bar{q}}\left(\frac{2 \bar{q}^{3}}{1-\bar{q}}\left(u_{j}-1\right)-\frac{2 \bar{q}^{3} q(1-q)}{(\bar{q}-q)^{2}(1-\bar{q})}\right)^{\frac{1}{2}} .
$$

Under this setting and introducing the parameter $\tau_{j}$ such that

$$
e^{\tau_{j}}=D_{G}\left(u_{j}\right)
$$

we get the theorem as follows.

\section{Theorem 2-4}

$$
\lim _{M \rightarrow \infty} \operatorname{Prob}\left(L\left(t_{1}, M\right) \geq \ell_{1}, L\left(t_{2}, M\right) \geq \ell_{2}, \cdots, L\left(t_{m}, M\right) \geq \ell_{m}\right)=\operatorname{det}\left(1+\mathcal{K}_{G} \mathcal{G}\right)
$$

where the Fredholm determinant and $\mathcal{G}\left(\tau_{j}, \xi\right)$ are defined in (3.26) and (3.27) respectively and

$$
\mathcal{K}_{G}\left(\tau_{1}, \xi_{1} ; \tau_{2}, \xi_{2}\right)= \begin{cases}\frac{\exp \left(-\xi_{1}^{2}\right)}{\sqrt{\pi}}-\frac{\exp \left(-\frac{\left(\xi_{2}-e^{\tau_{1}-\tau_{2}} \xi_{1}\right)^{2}}{\left.1-e^{2\left(\tau_{1}-\tau_{2}\right)}\right)}\right.}{\sqrt{\pi\left(1-e^{2\left(\tau_{1}-\tau_{2}\right)}\right)}}, & \text { for } \tau_{1}<\tau_{2} \\ \frac{\exp \left(-\xi_{1}^{2}\right)}{\sqrt{\pi}}, & \text { for } \tau_{1} \geq \tau_{2}\end{cases}
$$

This kernel represents the propagation of a Brownian particle (a particle obeying the 
Ornstein-Uhlenbeck process). Actually the two point distribution is calculated as

$$
\begin{aligned}
& \lim _{M \rightarrow \infty} \operatorname{Prob}\left(L\left(t_{1}, M\right) \geq \ell_{1}, L\left(t_{2}, M\right) \geq \ell_{2}\right) \\
& =1-\int_{s_{1}}^{\infty} d \xi_{1} \mathcal{K}_{n}\left(\tau_{1}, \xi_{1} ; \tau_{1}, \xi_{1}\right)-\int_{s_{2}}^{\infty} d \xi_{2} \mathcal{K}_{n}\left(\tau_{2}, \xi_{2} ; \tau_{2}, \xi_{2}\right) \\
& +\frac{1}{2} \int_{s_{1}}^{\infty} d \xi_{1} \int_{s_{2}}^{\infty} d \xi_{2}\left|\begin{array}{ll}
\mathcal{K}_{n}\left(\tau_{1}, \xi_{1} ; \tau_{1}, \xi_{1}\right) & \mathcal{K}_{n}\left(\tau_{1}, \xi_{1} ; \tau_{2}, \xi_{2}\right) \\
\mathcal{K}_{n}\left(\tau_{2}, \xi_{2} ; \tau_{1}, \xi_{1}\right) & \mathcal{K}_{n}\left(\tau_{2}, \xi_{2} ; \tau_{2}, \xi_{2}\right)
\end{array}\right| \\
& +\frac{1}{2} \int_{s_{1}}^{\infty} d \xi_{1} \int_{s_{2}}^{\infty} d \xi_{2}\left|\begin{array}{ll}
\mathcal{K}_{n}\left(\tau_{2}, \xi_{2} ; \tau_{2}, \xi_{2}\right) & \mathcal{K}_{n}\left(\tau_{2}, \xi_{2} ; \tau_{1}, \xi_{1}\right) \\
\mathcal{K}_{n}\left(\tau_{1}, \xi_{1} ; \tau_{2}, \xi_{2}\right) & \mathcal{K}_{n}\left(\tau_{1}, \xi_{1} ; \tau_{1}, \xi_{1}\right)
\end{array}\right| \\
& =\int_{-\infty}^{s_{1}} d \xi_{1} \int_{-\infty}^{s_{2}} d \xi_{2} \frac{e^{\frac{-\left(\xi_{2}-e^{\tau_{1}-\tau_{2}} \xi_{1}\right)^{2}}{1-e^{2\left(\tau_{1}-\tau_{2}\right)}}}}{\sqrt{\pi\left(1-e^{2\left(\tau_{1}-\tau_{2}\right)}\right)}} \frac{e^{-\xi_{1}^{2}}}{\sqrt{\pi}}
\end{aligned}
$$

Next we consider the case $\bar{q}_{1} \sim \bar{q}_{2} \sim \cdots \sim \bar{q}_{n}$. The typical picture of the time evolution of the particles is depicted in Fig. 2(c). In this figure, it seems that four groups of which each top particle is the defect particle are formed and each group behaves like one particle in the TASEP. Thus in the region, one can expect that the system is effectively the same as $n$ TASEP particles.

In addition to the scaling $(3.42)-(3.44)$, we also set

$$
\bar{q}_{i}=\bar{q}-\bar{q}(1-\bar{q}) \frac{2 \epsilon_{i}}{M^{\frac{1}{2}}} .
$$

Note that the scaling exponent $1 / 2$ in (3.42) is the same as that in the case of the central limit theorem. However the following theorem indicates that the limiting process of $L(t, M)$ depends on the number $n$ of the defect particles. Note that this is not the information which the scaling exponent can detect.

\section{Theorem 2-4'}

$$
\lim _{M \rightarrow \infty} \operatorname{Prob}\left(L\left(t_{1}, M\right) \geq \ell_{1}, L\left(t_{2}, M\right) \geq \ell_{2}, \cdots, L\left(t_{m}, M\right) \geq \ell_{m}\right)=\operatorname{det}\left(1+\mathcal{K}^{(n)} \mathcal{G}\right) .
$$

The Fredholm determinant and $\mathcal{G}\left(\tau_{j}, \xi\right)$ in the right hand side are defined in (3.26) and (3.27) respectively and the kernel is given by

$$
\mathcal{K}^{(n)}\left(\tau_{1}, x_{1} ; \tau_{2}, x_{2}\right)=\tilde{K}^{(n)}\left(\tau_{1}, x_{1} ; \tau_{2}, x_{2}\right)-\phi\left(\tau_{1}, x_{1} ; \tau_{2}, x_{2}\right),
$$

where

$$
\begin{aligned}
& \tilde{K}^{(n)}\left(\tau_{1}, \xi_{1} ; \tau_{2}, \xi_{2}\right)=\frac{2}{(2 \pi i)^{2}} \int_{\Gamma} d w_{1} \int_{\gamma} d w_{2} \frac{\exp \left(w_{2}^{2}-w_{1}^{2}-2 w_{2} \xi_{2}+2 w_{1} \xi_{1}\right)}{e^{\tau_{1}-\tau_{2}} w_{2}-w_{1}} \prod_{j=1}^{n} \frac{e^{-\tau_{2}} w_{2}+\epsilon_{j}}{e^{-\tau_{1}} w_{1}+\epsilon_{j}} \\
& \phi\left(\tau_{1}, \xi_{1} ; \tau_{2}, \xi_{2}\right)= \begin{cases}\frac{1}{\sqrt{\pi\left(1-e^{2\left(\tau_{1}-\tau_{2}\right)}\right)}} \exp \left(-\frac{\left(x_{2}-e^{\tau_{1}-\tau_{2}} \xi_{1}\right)^{2}}{1-e^{2\left(\tau_{1}-\tau_{2}\right)}}\right), & \text { for } \tau_{1}<\tau_{2}, \\
0, & \text { for } \tau_{1} \geq \tau_{2} .\end{cases}
\end{aligned}
$$


In (3.51), $\Gamma$ represents the contour enclosing $-e^{\tau_{1}} \epsilon_{i}(i=1, \cdots, n)$ anticlockwise and $\gamma$ represents the arbitrary path running from $-i \infty$ to $i \infty$.

The kernel above has been given in $[55,59]$. As in Theorem 2-3', it is reduced to $\mathcal{K}_{G}$ (3.46) if we set $\epsilon=0$ for some $j$ and $\epsilon_{k}=\infty$ for $k \neq j$. Thus, later we will give only the proof of this theorem in Section 5.3.

The Fredholm determinant also describes the joint distribution function of the largest eigenvalue in the Hermitian multi-matrix model (3.39), where we assume the rank of $H_{j}(j=1, \cdots, m)$ is $n$ and $V=\operatorname{diag}\left(\epsilon_{1}, \epsilon_{2}, \cdots, \epsilon_{n}\right)$. The joint distribution of the largest eigenvalues $l^{(i)}$ of $H_{i}$ can be described as the Fredholm determinant in the above theorem,

$$
\operatorname{Prob}\left(l^{(1)} \leq s_{1}, \cdots, l^{(m)} \leq s_{m}\right)=\operatorname{det}\left(1+\mathcal{K}^{(n)} \mathcal{G}\right) .
$$

Note that in the equation above, we do not take the limit as the rank $n$ goes to infinity.

\subsection{Scaling limit 2 ( $M$ fixed)}

Here we consider another scaling limit such that $M$ is fixed and $t$ goes to infinity. We set

$$
\begin{aligned}
t_{i} & =e^{2 \tau_{i}} T, \\
\ell_{i} & =(1-q) t_{i}-s_{i} \sqrt{2 q(1-q) t_{i}} . \\
q_{i} & =q-\sqrt{\frac{2 q(1-q)}{T}} \epsilon_{i},
\end{aligned}
$$

and consider the asymptotic behavior as $T$ goes to infinity. We get the following theorem. The proof of the theorem will be given in Section 5.4.

\section{Theorem 3}

$$
\lim _{T \rightarrow \infty} \operatorname{Prob}\left(L\left(t_{1}, M\right) \geq \ell_{1}, L\left(t_{2}, M\right) \geq \ell_{2}, \cdots, L\left(t_{m}, M\right) \geq \ell_{m}\right)=\operatorname{det}\left(1+\mathcal{K}^{(M)} \mathcal{G}\right),
$$

where the right hand side is the same as that of (3.49) with $n=M$.

\subsection{Continuous limit}

In this subsection, we consider the continuous time version of the TASEP which is also usually discussed in the study of the ASEP. In the case without defect particles, the rule is defined as follows. Let $\tilde{t}$ be the time variable which can take any positive real number. Between time $\tilde{t}$ and $\tilde{t}+d \tilde{t}$, a particle can hop to its right neighboring site with probability $d \tilde{t}$ if the site is empty. If the site is occupied, the particle stays at the same site with probability 1.

We easily find that the continuous time version can be realized from the discrete time version defined in Section 2 by taking the limit,

$$
1-q \rightarrow 0, t \rightarrow \infty
$$


with $\tilde{t}=(1-q) t$ fixed. In addition, the scaling limit in Section 3.2 can also be studied by taking the $\tilde{t} \rightarrow \infty, M \rightarrow \infty$ limit with their ratio

$$
\tilde{u}=\tilde{t} / M
$$

fixed. Applying (3.58) and (3.59) to the Theorems in Section 3.2, we can rewrite the theorems for the continuous time TASEP. Here we show only the result which corresponds to Theorem 2-2. Analogous to (3.21) and (3.22), we scale time $\tilde{t}_{j}$ and the position $\ell_{j}$ as

$$
\begin{aligned}
& \tilde{t}_{j}=\tilde{u} M+C^{(c)}(\tilde{u}) M^{\frac{2}{3}} \tau_{j}, \\
& \ell_{j}=A_{2}^{(c)}\left(\tilde{u}_{j}\right) M-D^{(c)}(\tilde{u}) M^{\frac{1}{3}} s_{i},
\end{aligned}
$$

where $1<\tilde{u}$ and

$$
\begin{aligned}
& A_{2}^{(c)}(\tilde{u})=(\sqrt{\tilde{u}}-1)^{2}, \\
& C^{(c)}(\tilde{u})=2 \tilde{u}^{\frac{5}{6}}(\sqrt{\tilde{u}}-1)^{\frac{1}{3}}, \\
& D^{(c)}(\tilde{u})=\tilde{u}^{\frac{1}{6}}(\sqrt{\tilde{u}}-1)^{\frac{2}{3}} .
\end{aligned}
$$

Then we have

$$
\lim _{M \rightarrow \infty} \operatorname{Prob}\left(L\left(\tilde{t}_{1}, M\right) \geq \ell_{1}, L\left(\tilde{t}_{2}, M\right) \geq \ell_{2}, \cdots, L\left(\tilde{t}_{m}, M\right) \geq \ell_{m}\right)=\operatorname{det}\left(1+\mathcal{K}_{2} \mathcal{G}\right) .
$$

Here the right hand side is defined in $(3.26)-(3.28)$.

\section{From the TASEP to the Schur process}

In this section, we discuss a relationship between the time evolution of a tagged particle in the TASEP with the step initial condition described in Section 2 and the stochastic growth of a Young diagram $[32,33,34,35]$ which turns out to be a special case of the Schur process $[36,37]$. In the last part of this section, the proof of Theorem 1 in Section 3.1 is obtained.

In the study of the TASEP, the technique using the enumeration of Young diagrams was used in [17] to study the current fluctuation. The discussion in [17] was based on the relation of the TASEP to the directed polymer problem on a matrix of which the elements obey the geometric distribution. In this section, on the other hand, we consider the correspondence between the directed polymer problem on a 01 matrix $[28,29,30,31]$ and the TASEP, which is a similar but different mapping. For the analysis of the tagged particle problem, the mapping is more natural and convenient. 


\subsection{TASEP and 01 matrix}

Let us consider an ensemble of $N \times M 01$ matrices $\{a(i, j)\}_{i=1, \cdots, N, j=1, \cdots, M}$, whose matrix elements are either 0 or 1 . All matrix elements are independent random variables and each element obeys the Bernoulli distribution,

$$
a(i, M+1-j)= \begin{cases}0, & \text { with probability } 1-q_{j}, \\ 1, & \text { with probability } q_{j} .\end{cases}
$$

Here $1-q_{j}$ is taken to be the same value as the hopping rate of the $j$ th particle of the TASEP defined in Section 2. Each realization of the 01 matrix $a(i, j)$ can be translated to a time evolution of the TASEP as follows.

$a(i, M+1-j)=0$ (resp. 1): Between time $i+j-2$ and $i+j-1$, the $j$ th particle tries to hop to the right neighboring site (resp. stays at the same site). When the right neighboring site is occupied at time $i+j-2$, particle can not move due to the exclusion effect even if $a(i, M+1-j)=0$.

Since $a(i, M+1-j)=0$ could mean both hopping or stay depending on whether the target site is occupied or empty, the mapping from a 01 matrix to the time evolution of the TASEP is not one-to-one. In Fig. 4, we depict an example of the time evolution in the TASEP and the corresponding two 01 matrices. Note that in the step initial condition, the $j$ th particle cannot move until time $j-1$ and that the time evolution of the $j$ th particle at time step between $i$ and $i+1$ does not depend on that of the $j-1$ th particle at the time step. Thus one finds that the 01 matrix defined above has all informations needed for the time evolution of the $M$ th particle from time 0 through $M+N-1$. by

Let us define a left-down path $\pi(N, M)$ of a $N \times M 01$ matrix $\{a(i, j)\}_{i=1, \cdots, N, j=1, \cdots, M}$

$$
\pi(N, M)=\left\{\left\{\left(i_{k}, j_{k}\right)\right\}_{1 \leq k} \mid a\left(i_{k}, j_{k}\right)=1,1 \leq i_{1}<i_{2}<\cdots \leq N, M \geq j_{1} \geq j_{2} \geq \cdots \geq 1\right\} .
$$

Note that the row indices $i_{k}(k=1, \cdots)$ are strictly increasing while the column indices $j_{k}(k=1, \cdots)$ are weakly decreasing. In $\pi$, the position of $\left(i_{k+1}, j_{k+1}\right)$ is always located on the down side or left-down side of the position of $\left(i_{k}, j_{k}\right)$ in the 01 matrix. We also define the quantity $G(N, M)$ by

$$
G(N, M)=\max _{\pi(N, M)}|\pi(N, M)|,
$$

where $|\pi|$ denotes the number of elements in the path $\pi$. If we regard the 01 matrix as the random media in a plane, $\pi(N, M)$ as a spatial configuration of a polymer chain, and $|\pi(N, M)|$ as its energy, we can interpret (4.3) as a statistical mechanical problem of a directed polymer. This kind of problem is studied in [28, 29, 30, 31].

Now we find that this maximum length of the left-down path is directly related to the position of a tagged particle as

$$
G(N, M)=d(N, M)
$$


where

$$
d(N, M):=N-L(t=N+M-1, M)
$$

represents the number of times the $M$ th particle stays at the same site from time 0 through $N+M-1$. The equation (4.4) enables us to study the dynamics of the TASEP in terms of the 01 matrices and plays a fundamental role in our subsequent analysis.

We can show (4.4) in the following way. For the case $M=1$ (the case where we tag the first particle), we can easily check this equation, since $G(N, 1)$ defined in (4.3) is simply the total number of the value 1 in the sequence $\{a(k, 1)\}_{k=1, \cdots, N}$ and it is clear that this quantity represents the number of times the particle stays from $t=0$ through $N$.

For the case $M \geq 2$, we can prove (4.4) by mathematical induction about the row $N$. Here, we mainly consider only the case $M=2$. For the case $M \geq 3$, we can check (4.4) in a similar fashion to this case.

When $N=1$, we can check (4.4) easily for all four cases $(a(1,1)=0,1$ and $a(1,2)=$ $0,1)$.

We assume that (4.4) holds for $N=N_{a}$, and $M=1$ and 2, i.e.,

$$
d\left(N_{a}, 1\right)=G\left(N_{a}, 1\right), \quad d\left(N_{a}, 2\right)=G\left(N_{a}, 2\right) .
$$

Under this assumption, we will show that $d\left(N_{a}+1,2\right)=G\left(N_{a}+1,2\right)$ as follows. From (4.3), one easily finds

$$
G\left(N_{a}, 2\right) \geq G\left(N_{a}, 1\right), \quad G\left(N_{a}, i\right) \geq G\left(N_{b}, i\right),
$$

where $1 \leq N_{b} \leq N_{a}$, and $i=1$ and 2 . Considering these properties, we classify the problem into two cases,

(i)

$$
G\left(N_{a}, 2\right)=G\left(N_{a}, 1\right), \text { and } a\left(N_{a}+1,2\right)=1,
$$

(ii)

$$
\left\{\begin{array}{l}
G\left(N_{a}, 2\right)>G\left(N_{a}, 1\right) \text { and } a(N+1,2)=0 \text { or } 1 \\
G\left(N_{a}, 2\right)=G\left(N_{a}, 1\right) \text { and } a(N+1,2)=0
\end{array}\right.
$$

The case (i) corresponds to the situation where the two particles occupy the neighboring sites at $t=N_{a}+1$. Thus the second particle (the tagged particle) must stay at time step between $N_{a}+1$ and $N_{a}+2$. Hence one finds

$$
d\left(N_{a}+1,2\right)=d\left(N_{a}, 2\right)+1,
$$

regardless of the value of $a\left(N_{a}+1,1\right)$. On the other hand, due to (4.3), (4.7) and (4.8), we have

$$
G\left(N_{a}+1,2\right)=G\left(N_{a}, 1\right)+a\left(N_{a}+1,2\right)=G\left(N_{a}, 2\right)+1 .
$$

Thus from (4.6), (4.10) and (4.11), we have for the case (i),

$$
d\left(N_{a}+1,2\right)=G\left(N_{a}+1,2\right) .
$$


The case (ii) corresponds to the situation where at $t=N_{a}+1$, the distance between the first and the second particles is at least one site. Note that in this case we do not need to consider the exclusion effect of the first particle and thus the situation is essentially the same as the case $M=1$. We have

$$
d\left(N_{a}+1,2\right)=d\left(N_{a}, 2\right)+a\left(N_{a}+1,1\right) .
$$

On the other hand, from (4.7) and (4.9) we can also check that

$$
G\left(N_{a}+1,2\right)=G\left(N_{a}, 2\right)+a\left(N_{a}+1,1\right) .
$$

Thus we find that (4.12) holds also for the case (ii) and therefore (4.4) holds for $M=2$.

In the case $M=k(k \geq 3)$, we can also classify the situation into two cases as in (i) and (ii) for $M=2$, i.e., the case (i)' where all $k$ particles are packed and (ii)' where the particles form several groups which are separated by some successive empty sites. For the case (i)', we can check (4.4) in a similar manner to the case $M=2$. For the case (ii)', we can easily show that the case is essentially the same as the case (i)' for $M=m$ which is smaller than $k$.

\subsection{1 matrix and the dual Robinson-Schensted-Knuth corre- spondence}

The quantity we would like to discuss is the multi-time distribution of $L(t, M)$,

$$
\operatorname{Prob}_{\mathrm{TASEP}}\left(L\left(t_{1}, M\right) \geq \ell_{1}, L\left(t_{2}, M\right) \geq \ell_{2}, \cdots, L\left(t_{m}, M\right) \geq \ell_{m}\right),
$$

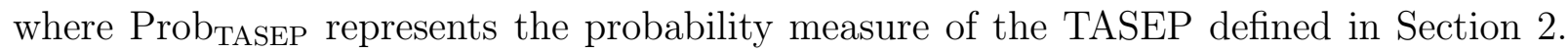
Due to the result in Section 4.1(especially (4.4) and (4.5)), one finds

$$
\begin{aligned}
& \operatorname{Prob}_{\mathrm{TASEP}}\left(L\left(N_{1}+M-1, M\right) \geq \ell_{1},\right. \\
& \left.\qquad\left(N_{2}+M-1, M\right) \geq \ell_{2}, \cdots, L\left(N_{m}+M-1, M\right) \geq \ell_{m}\right) \\
& =\operatorname{Prob}_{01}\left(G\left(N_{1}, M\right) \leq N_{1}-\ell_{1}, G\left(N_{2}, M\right) \leq N_{2}-\ell_{2}, \cdots, G\left(N_{m}, M\right) \leq N_{m}-\ell_{m}\right),
\end{aligned}
$$

where Prob $_{01}$ represents the probability measure of 01 table defined in (4.1) and $G\left(N_{i}, M\right)$ is defined in (4.4).

In this section, we further restate our problem in terms of the combinatorics of Young tableaux. For the definitions and basic properties of Young tableaux and related subjects, we refer the readers to [60]. There is a bijective mapping between an $N \times M 01$ matrix and a pair $(P, Q)$ where $P^{t}$ (the transpose of $P$ ) and $Q$ are the semistandard Young tableaux (SSYT) with the condition that the shape of $P$ is the same as that of $Q$. The mapping is called the dual Robinson-Schensted-Knuth (RSK) algorithm. In order to get $(P, Q)$, we first construct a two-line array (or generalized permutation),

$$
\left(\begin{array}{cccccccccc}
1 & \cdots & 1 & 2 & \cdots & 2 & \cdots & N & \cdots & N \\
j_{1}^{(1)} & \cdots & j_{m_{1}}^{(1)} & j_{1}^{(2)} & \cdots & j_{m_{2}}^{(2)} & \cdots & j_{1}^{(N)} & \cdots & j_{m_{N}}^{(N)}
\end{array}\right)
$$


where $1 \leq j_{1}^{(i)} \leq j_{2}^{(i)} \cdots \leq M$, from the 01 matrix by listing $\left(\begin{array}{l}i \\ j\end{array}\right)$ 's for which $a(i, j)=1$. Next, we construct $P$ (resp. $Q$ ) by arranging on a plane the figures in the second row (resp. the first row). Note that each positive integer constructing $P$ (resp. $Q$ ) is less than $M($ resp. $N)$. For details of the algorithm, see [60]. The generalized permutation and $(P, Q)$ corresponding to the 01 table in Fig. 5(b) are given in Fig. 6.

Now we express $G(N, M)(4.3)$ as the quantity related to a pair $(P, Q)$. Let us denote the shape of $P$ (or $Q$ ) by the Young diagram, $\lambda(N, M)=\left(\lambda_{1}(N, M), \lambda_{2}(N, M), \cdots\right)$ where $\lambda_{i} \geq 0$ represents the length of $i$ th row of the tableaux $P$ (or $Q$ ). In the example of Fig. $6(\mathrm{~b})$, we see $\lambda=(4,3,2,2,2)$. Then we have

$$
G(N, M)=\lambda_{1}^{\prime}(N, M) .
$$

Here $\lambda^{\prime}=\left(\lambda_{1}^{\prime}, \lambda_{2}^{\prime}, \ldots\right)$ means the transpose of the Young diagram $\lambda$. Thus $\lambda_{1}^{\prime}(N, M)$ is equal to the length of the first column of $\lambda(N, M)$.

Eq. (4.18) may be shown by examining the dual RSK algorithm directly but is also understood as follows. For the sequence of the numbers $\left(i_{1}, i_{2}, \cdots\right)$ where $i \in(0,1,2, \cdots)$, we define a nondecreasing (resp. nonincreasing) subsequence $\left(j_{1}, j_{2}, \cdots\right)$ such that $j_{1} \leq$ $j_{2} \leq \cdots$ (resp. $j_{1} \geq j_{2} \geq \cdots$ ). From the construction it is not difficult to see that $G(N, M)$ is equivalent to the length of the longest nonincreasing subsequence of the second row $\left(j_{1}^{(1)}, \cdots, j_{m_{1}}^{(1)}, \cdots, j_{1}^{(N)}, \cdots, j_{m_{N}}^{(N)}\right)$ in a generalized permutation $(4.17)$. Thus, $G(N, M)$ is also regarded as the length of the longest nondecreasing subsequence of the opposite sequence $\left(j_{m_{N}}^{(N)}, \cdots, j_{1}^{(N)}, \cdots, j_{m_{1}}^{(1)}, \cdots, j_{1}^{(1)}\right)$ of the second row in (4.17). We can apply the (normal) RSK algorithm [60] to this reverse sequence to obtain a SSYT. Let us denote the SSYT by $R$. Then (4.18) is a consequence of the facts that the length of the longest nondecreasing sequence in the reverse sequence is equal to the length of the first row of $R$ (this is known as a property of the normal RSK algorithm $[17,20]$ ) and the symmetry property,

$$
P^{t}=R,
$$

whose proof can be found in Appendix A of [61]. Notice that in the example of Fig. 5(a), $L(9,4)=1$ thus $d(6,4)=5$ while in Fig. 6(b), $\lambda_{1}^{\prime}=5$.

Thus from (4.18), we get

$$
\begin{aligned}
& \operatorname{Prob}_{01}\left(G\left(N_{1}, M\right) \leq N_{1}-\ell_{1}, G\left(N_{2}, M\right) \leq N_{2}-\ell_{2}, \cdots, G\left(N_{m}, M\right) \leq N_{m}-\ell_{m}\right) \\
& =\operatorname{Prob}_{\lambda}\left(\lambda_{1}^{\prime}\left(N_{1}, M\right) \leq N_{1}-\ell_{1}, \lambda_{1}^{\prime}\left(N_{2}, M\right) \leq N_{2}-\ell_{2}, \cdots, \lambda_{1}^{\prime}\left(N_{m}, M\right) \leq N_{m}-\ell_{m}\right) .
\end{aligned}
$$

Here $\operatorname{Prob}_{\lambda}$ in the right hand side represents the probability measure of the set of Young diagrams obtained by the dual RSK algorithm from the 01 matrix defined in (4.1) and $\lambda\left(N_{i}, M\right)$ means the Young diagram obtained by applying the dual RSK algorithm to the $N_{i} \times M 01$ submatrix $\{a(i, j)\}_{1 \leq i \leq N_{i}, 1 \leq j \leq M}$ of the $N \times M 01$ matrix (4.1).

From (4.16) and (4.20), we managed to interpret the time evolution of a tagged particle in the TASEP as the time evolution of $\lambda_{1}^{\prime}$, the length of the first column, regarding $N_{i}$ as a time parameter. 


\subsection{Growth of Young diagrams and Schur process}

In order to investigate the right hand side of (4.20), we consider the following probability,

$$
\operatorname{Prob}_{\lambda}\left(\lambda(1, M)=\lambda^{(1)}, \lambda(2, M)=\lambda^{(2)}, \cdots, \lambda(N, M)=\lambda^{(N)}=\lambda\right)
$$

for a given set of Young diagrams, $\left\{\lambda^{(k)}\right\}_{k=1, \ldots, N}$. This equation describes the growth of a Young diagram. Eq. (4.21) represents the joint distribution of them. Fig. 7 illustrates the growth of Young diagram which corresponds to Fig. 5(b). Notice that from (4.4) and (4.18), the growth of $\lambda_{1}^{\prime}(i, M)$ describes the time evolution of $M$ th particle in the TASEP. Let us denote the $Q^{(k)}$ as the part of the $Q$ tableau obtained by picking up only the symbols $1,2, \cdots, i$. From the rule of the dual RSK algorithm [60], we find that $Q^{(k)}$ is equivalent to the $Q$ tableau obtained by applying the dual RSK algorithm to the the submatrix $\left\{a(i, j)_{i=1, \ldots, k, j=1, \ldots, M}\right\}$. Thus one has

$$
\operatorname{sh}\left(Q^{(i)}\right)=\lambda(i, M)
$$

where $\operatorname{sh}\left(Q^{(i)}\right)$ denotes the shape of $Q^{(i)}$. Hence the $Q$ tableau records the growth of Young diagram, that is, it one-to-one corresponds to the set of the Young diagrams $\{\lambda(i, M)\}_{i=1, \cdots, N}$. Compare the tableau $Q$ in Fig. 6(b) with $\{\lambda(i, M)\}_{i=1, \cdots, 6}$ in Fig. 7. We can recognize that $\operatorname{sh}\left(Q^{(i)}\right)$ constructed from the tableau $Q$ in Fig. 6(b) is equivalent to $\lambda(i, M)$ in Fig. 7 . Hence we have

$$
\begin{aligned}
& \operatorname{Prob}_{\lambda}\left(\lambda(1, M)=\lambda^{(1)}, \lambda(2, M)=\lambda^{(2)}, \cdots, \lambda(N, M)=\lambda^{(N)}\right) \\
& =\operatorname{Prob}_{\{P, Q\}}\left(\operatorname{sh}\left(Q^{(k)}\right)=\lambda^{(k)}, k=1, \ldots, N\right) .
\end{aligned}
$$

Here $\operatorname{Prob}_{\{P, Q\}}$ represents the probability measure on the set of the pairs $\{P, Q\}$ obtained by the dual RSK algorithm from the $N \times M 01$ matrices defined in (4.1). Hence it is found that the growth process of the Young diagram is characterized by the $Q$ tableau in the dual RSK correspondence. This type of growth process has been also discussed in $[32,33,34,35]$.

Remembering $P$ is constructed from the second row of a generalized permutation, we find that the symbol $j$ in $P$ represents the column index of the element of the 01 matrix where $a(i, j)=1$. Thus the total number of $j \mathrm{~s}$ in $P$ is equal to the total number of the figure 1 in the $j$ th column of the 01 matrix. Hence, for a given $P^{\prime}$, one finds

$$
\begin{aligned}
& \operatorname{Prob}_{\{P, Q\}}\left(P=P^{\prime}, \operatorname{sh}\left(Q^{(k)}\right)=\lambda^{(k)}(k=1, \cdots, N)\right) \\
& =\left\{\begin{array}{l}
q_{M}^{\sharp 1\left(P^{\prime}\right)} q_{M-1}^{\sharp 2\left(P^{\prime}\right)} \cdots q_{1}^{\sharp M\left(P^{\prime}\right)} \times\left(1-q_{M}\right)^{N-\sharp 1\left(P^{\prime}\right)}\left(1-q_{M-1}\right)^{N-\sharp 2\left(P^{\prime}\right)} \cdots\left(1-q_{1}\right)^{N-\sharp M\left(P^{\prime}\right),} \\
\text { if } \lambda^{(1)} \text { and } \lambda^{(i+1)} / \lambda^{(i)}(i=1, \cdots, N-1) \text { have no two squares in the same column, } \\
0, \text { otherwise. }
\end{array}\right.
\end{aligned}
$$

where $\sharp i\left(P^{\prime}\right)$ means the total number of the symbol $i$ in $P^{\prime}$. Applying the above equation to the combinatorial definition of the Schur function,

$$
s_{\lambda / \mu}\left(a_{1}, \cdots, a_{N}\right)=\sum_{S} a_{1}^{\sharp 1(S)} \cdots a_{N}^{\sharp N(S)},
$$


where the summation is taken to all $\operatorname{SSYT} S$ with shape $\lambda / \mu$, we have

$$
\begin{aligned}
& \operatorname{Prob}_{\{P, Q\}}\left(\operatorname{sh}\left(Q^{(k)}\right)=\lambda^{(k)}, k=1, \ldots, N\right) \\
& =\sum_{P^{\prime}} \operatorname{Prob}_{\{P, Q\}}\left(P=P^{\prime}, \operatorname{sh}\left(Q^{(k)}\right)=\lambda^{(k)}, k=1, \ldots, N\right) \\
& =\left(1-q_{1}\right)^{N} \cdots\left(1-q_{M}\right)^{N} s_{\lambda^{\prime}(N)}\left(p_{M}, \cdots, p_{1}\right) \\
& \quad s_{\lambda^{(1)}}(1,0, \cdots) s_{\lambda^{(2)} / \lambda^{(1)}}(1,0, \cdots) \times \cdots \times s_{\lambda^{(N)} / \lambda^{(N-1)}}(1,0, \cdots),
\end{aligned}
$$

where $p_{i}=q_{i} /\left(1-q_{i}\right)$. The factor $s_{\lambda^{(1)}}(1,0, \cdots) \cdots$ ensures that the probability is zero unless $\lambda^{(1)}$ and $\lambda^{(i+1)} / \lambda^{(i)}(i=1, \cdots, N-1)$ have no two squares in the same column. Thus combining (4.23) with (4.26), we finally find

$$
\begin{aligned}
& \operatorname{Prob}_{\lambda}\left(\lambda(1, M)=\lambda^{(1)}, \lambda(2, M)=\lambda^{(2)}, \cdots, \lambda(N, M)=\lambda^{(N)}\right) \\
& =\left(1-q_{1}\right)^{N} \cdots\left(1-q_{M}\right)^{N} s_{\lambda^{(N)}}\left(p_{M}, \cdots, p_{1}\right) \times \\
& s_{\lambda^{(1)}}(1,0, \cdots) s_{\lambda^{(2)} / \lambda^{(1)}}(1,0, \cdots) \times \cdots \times s_{\lambda^{(N)} / \lambda^{(N-1)}}(1,0, \cdots) .
\end{aligned}
$$

This measure is the special case of the joint distribution function in the Schur process [36, 37]. Since the Schur function is expressed as a determinant, the function (4.27) is described as a product of determinants. Furthermore, it is known that the process has a remarkable mathematical structure that the correlation function of $\left\{\lambda_{i}^{(j)}\right\}_{i=1,2, \cdots, j=1, \cdots N}$ can be expressed as a determinant and its scaling limit can be discussed exactly. The Schur process also appears in other fields of physics and mathematics such as random growth process [38], melting problem of a three-dimensional crystal [62], random tiling model [63], and so on.

\subsection{Proof of Theorem 1}

From (4.16),(4.20) and (4.27), we find

$$
\begin{aligned}
& \operatorname{Prob}_{\mathrm{TASEP}}\left(L\left(t_{1}, M\right) \geq \ell_{1}, L\left(t_{2}, M\right) \geq \ell_{2}, \cdots, L\left(t_{m}, M\right) \geq \ell_{m}\right) \\
& =\operatorname{Prob}_{\mathrm{SP}}\left(\lambda_{1}^{\prime}\left(N_{1}, M\right) \leq N_{1}-\ell_{1}, \lambda_{1}^{\prime}\left(N_{2}, M\right) \leq N_{2}-\ell_{2}, \cdots, \lambda_{1}^{\prime}\left(N_{m}, M\right) \leq N_{m}-\ell_{m}\right) .
\end{aligned}
$$

Here ProbsP represents the probabilistic measure of the Schur process (4.26).

In general, the Schur process is defined as follows. For the set of the Young diagrams such that

$$
\lambda^{(1)} \supset \mu^{(1)} \subset \lambda^{(2)} \supset \mu^{(2)} \cdots \supset \mu^{(N-1)} \subset \lambda^{(N)},
$$

the following weight is assigned

$$
s_{\lambda^{(1)}}\left(\rho_{0}^{+}\right) s_{\lambda^{(1)} / \mu^{(1)}}\left(\rho_{1}^{-}\right) s_{\lambda^{(2)} / \mu^{(1)}}\left(\rho_{1}^{+}\right) \cdots s_{\lambda^{(N)} / \mu^{(N-1)}}\left(\rho_{N-1}^{+}\right) s_{\lambda^{(N)}}\left(\rho_{N}^{-}\right) .
$$

Here $s_{\rho / \mu}(\rho)$ is the Schur function with the specialization of algebra $\rho$. In our case, we notice only two cases

$$
s_{\lambda / \mu}(\rho)= \begin{cases}s_{\lambda / \mu}\left(a_{1}, \cdots, a_{N}\right), & \text { for } \rho=\rho(z)=\prod_{j=1}^{N} \frac{1}{1-a_{j} z} \\ s_{\lambda^{\prime} / \mu^{\prime}}\left(a_{1}, \cdots, a_{N}\right), & \text { for } \rho=\rho(z)=\prod_{j=1}^{N}\left(1+a_{j} z\right)\end{cases}
$$


where $s_{\lambda / \mu}\left(a_{1}, \cdots, a_{N}\right)$ is defined in (4.25). Substituting to (4.29),

$$
\begin{aligned}
& \rho_{i}^{-}(z)=1+z, \quad(1 \leq i \leq N-1) \\
& \rho_{N}^{-}=\prod_{i=1}^{M} \frac{1}{1-p_{i} z} \\
& \rho_{j}^{+}(z)=1, \quad(0 \leq j \leq N-1)
\end{aligned}
$$

where $p_{i}=q_{i} /\left(1-q_{i}\right)$ and applying Theorem 1 in [36] or Theorem 2.2 in [37] to this case, we finally obtain the Fredholm determinant representation((3.3)-(3.7)) of (4.28).

\section{$5 \quad$ Asymptotics}

In this section, we discuss the scaling limit of the multi-time distribution function (2.1) by applying the saddle point method to the kernel (3.5)-(3.7) in Theorem 1.

We consider two types of scaling limit. The first case is explained in Section 3.2. We focus on the situation where both time $t$ and the label of the tagged particle $M$ tend to infinity when there are $n$ defect particles with the stay rates $\left\{\bar{q}_{j}\right\}_{j=1, \cdots, n}$ in front of the tagged particle. The results on the limiting distributions are summarized as Theorem 2. Their proofs are given in Section 5.1, 5.2 and 5.3.

In the second case, we take $t$ to be infinite with $M$ fixed. The limiting distribution is described in Theorem 3 in Section 3.3, whose proof is given in Section 5.4.

\subsection{Region 1 (proof of Theorem 2-1)}

In this section, we consider the scaling limit in the region 1 in Fig. 3 . We scale time $t_{i}$ as (3.14). In order to calculate the limiting distribution, we rewrite the kernel (3.5)-(3.7) with the condition (3.9) as

$$
\begin{aligned}
& K\left(t_{1}, x_{1} ; t_{2}, x_{2}\right) \\
& =\tilde{K}\left(t_{1}, x_{1} ; t_{2}, x_{2}\right)-\phi_{t_{1}, t_{2}}\left(x_{1}, x_{2}\right) \\
& =\frac{1}{(2 \pi i)^{2}} \int_{C_{R_{1}^{\prime}}} \frac{d z_{1}}{z_{1}} \int_{C_{R_{2}^{\prime}}} \frac{d z_{2}}{z_{2}} \frac{z_{1}}{z_{1}-z_{2}} \frac{\left(1+1 / z_{2}\right)^{t_{2}-M+1}}{\left(1+1 / z_{1}\right)^{t_{1}-M+1}}\left(\frac{1-p z_{2}}{1-p z_{1}}\right)^{M-n} \prod_{i=1}^{n} \frac{1-\bar{p}_{i} z_{2}}{1-\bar{p}_{i} z_{1}} \frac{z_{2}^{x_{2}}}{z_{1}^{x_{1}}},
\end{aligned}
$$

where $p=q /(1-q)$ and $\bar{p}_{i}=\bar{q}_{i} /\left(1-\bar{q}_{i}\right) . C_{R}$ denotes the contour with radius $R$ enclosing the origin anticlockwise and $R_{i}^{\prime}(i=1,2)$ satisfy the condition, $1<R_{2}^{\prime}<R_{1}^{\prime}<1 / \bar{p}_{i}$ for $t_{1} \geq t_{2}$ and $1<R_{1}^{\prime}<R_{2}^{\prime}<1 / \bar{p}_{i}$ for $t_{1}<t_{2}$. Using (5.1) and the relation

$$
\frac{z_{1}}{z_{1}-z_{2}}= \begin{cases}\sum_{m=0}^{\infty}\left(\frac{z_{2}}{z_{1}}\right)^{m}, & t_{1} \geq t_{2}, \\ -\sum_{m=0}^{\infty}\left(\frac{z_{1}}{z_{2}}\right)^{m+1}, & t_{1}<t_{2}\end{cases}
$$


one finds

$$
K\left(t_{1}, x_{1} ; t_{2}, x_{2}\right)= \begin{cases}\sum_{m=0}^{\infty} \Psi_{1}\left(x_{1}+m, t_{1}\right) \Psi_{2}\left(x_{2}+m, t_{2}\right), & t_{1} \geq t_{2}, \\ -\sum_{m=0}^{\infty} \Psi_{1}\left(x_{1}-m-1, t_{1}\right) \Psi_{2}\left(x_{2}-m-1, t_{2}\right), & t_{1}<t_{2} .\end{cases}
$$

Here the function $\Psi_{1}\left(m, t_{1}, x_{1}\right)$ and $\Psi_{2}\left(m, t_{2}, x_{2}\right)$ are given by

$$
\begin{aligned}
& \Psi_{1}\left(x_{1}, t_{1}\right)=\frac{1}{2 \pi i} \int_{C_{R_{1}}} \frac{d z}{z} \frac{1}{(1+1 / z)^{t_{1}-M+1}(1-p z)^{M}} \prod_{i=1}^{n} \frac{1-p z}{1-\bar{p}_{i} z} \frac{1}{z^{x_{1}}}, \\
& \Psi_{2}\left(x_{2}, t_{2}\right)=\frac{1}{2 \pi i} \int_{C_{1}} \frac{d w}{w}(1+1 / w)^{t_{2}-M+1}(1-p w)^{M} \prod_{i=1}^{n} \frac{1-\bar{p}_{i} w}{1-p w} w^{x_{2}},
\end{aligned}
$$

where the radius $R_{1}$ of the contour $C_{R_{1}}$ in (5.4) is taken such that $1<R_{1}<1 / \bar{p}_{i}$. In the following discussion, we evaluate the asymptotics of the kernel (5.1) by applying the saddle point method to the functions $\Psi_{i}(x, t)(i=1,2)$.

First we consider the asymptotics of $\Psi_{1}\left(x_{1}, t_{1}\right)$. Substituting (3.14) with (5.4), we set

$$
\Psi_{1}\left(x_{1}, t_{1}\right)=\frac{1}{2 \pi i} \int_{C_{R_{1}}} \frac{d z}{z} e^{-M f(z)} \frac{1}{(1+z)^{1+M^{\frac{1}{2}} D_{1} \tau_{1}}} \prod_{i=1}^{n} \frac{1-p z}{1-\bar{p}_{i} z} \frac{1}{z^{x_{1}-t_{1}+M-1}},
$$

where

$$
f(z)=p \log (1+z)+\log (1-p z) .
$$

From the equation $f^{\prime}\left(z_{c}\right)=0$, we find the critical point $z_{c}$ is given by

$$
z_{c}=0
$$

Scaling the variable $z$ around the critical point as

$$
z=z_{c}+\frac{z^{\prime}}{D_{1} M^{\frac{1}{2}}}=\frac{z^{\prime}}{D_{1} M^{\frac{1}{2}}}
$$

where $D_{1}=\sqrt{q} /(1-q)=\sqrt{p(1+p)}$, we evaluate the asymptotics of the term $e^{-M f(z)}$ in (5.6) by the saddle point method,

$$
e^{-M f(z)} \sim e^{-M\left(f(0)+\frac{f^{\prime \prime}(0)}{2 D_{1}^{2} M} z^{\prime 2}\right)}=e^{\frac{z^{2}}{2}},
$$

as $M \rightarrow \infty$. Next we consider the asymptotics of other terms in (5.6). From (5.9), one obtains

$$
\frac{1}{(1+z)^{M^{\frac{1}{2}} D_{1} \tau_{1}+1}} \sim e^{-\tau_{1} z^{\prime}}, \prod_{i=1}^{n} \frac{1-p z}{1-\bar{p}_{i} z} \sim 1
$$

Transforming $x_{i}$ into $x_{i}^{\prime}(i=1,2)$,

$$
x_{i}=t_{i}-M+1-x_{i}^{\prime},
$$


and considering (5.9), one gets

$$
\frac{1}{z^{x_{1}-t_{1}+M-1}} \sim \frac{z^{x_{1}^{\prime}}}{D_{1}^{x_{1}^{\prime}} M^{\frac{x_{1}^{\prime}}{2}}} .
$$

Note that by the transformation (5.12), the function $g\left(t_{i}, x\right)(3.4)$ in the Fredholm determinant (3.3) is changed to $g\left(\tau_{i}, x^{\prime}\right)=-\chi_{\left(-\infty, \ell_{i}\right)}\left(x^{\prime}\right)$. From (5.9)-(5.13), we obtain the asymptotic form of $\Psi_{1}\left(x_{1}, t_{1}\right)$,

$$
\Psi_{1}\left(x_{1}, t_{1}\right) \sim \frac{D_{1}^{-x_{1}^{\prime}} M^{\frac{-x_{1}^{\prime}}{2}}}{2 \pi i} \int_{-i \infty+\epsilon}^{i \infty+\epsilon} d z^{\prime} e^{\frac{z^{\prime 2}}{2}-\tau_{1} z^{\prime}} z^{\prime x_{1}^{\prime}-1},
$$

where $\epsilon>0$.

Next we consider the asymptotics of $\Psi_{2}\left(x_{2}, t_{2}\right)$ (5.5). Similar to $\Psi_{1}\left(x_{1}, t_{1}\right)$, we set

$$
\Psi_{2}\left(x_{2}, t_{2}\right)=\frac{1}{2 \pi i} \int_{C_{1}} \frac{d w}{w} e^{M f(w)}(1+z)^{1+M^{\frac{1}{2}} D_{1} \tau_{2}} \prod_{i=1}^{n} \frac{1-\bar{p}_{i} w}{1-p w} w^{x_{2}-t+M-1} .
$$

Here $f(w)$ is defined in (5.7) and $C_{1}$ denotes the contour enclosing the origin anticlockwise with radius 1. Applying the saddle point method to this equation in the same way as $\Psi_{1}\left(x_{1}, t_{1}\right)$, we get

$$
\Psi_{2}\left(x_{2}, t_{2}\right) \sim \frac{D_{1}^{x_{2}^{\prime}} M^{\frac{x_{2}^{\prime}}{2}}}{2 \pi i} \int_{C_{1}} \frac{d w^{\prime}}{w^{\prime x_{2}^{\prime}+1}} e^{-\frac{w^{\prime 2}}{2}+\tau_{2} w^{\prime}} .
$$

Thus from (5.3), (5.14), and (5.16), we find

$$
\begin{aligned}
& \lim _{M \rightarrow \infty} K\left(t_{1}, x_{1} ; t_{2}, x_{2}\right) \\
& = \begin{cases}\left(D_{1} \sqrt{M}\right)^{x_{2}^{\prime}-x_{1}^{\prime}} \sum_{m=0}^{\infty} \psi_{1}\left(x_{1}^{\prime}-m, \tau_{1}\right) \psi_{2}\left(x_{2}^{\prime}-m, \tau_{2}\right), & \tau_{1} \geq \tau_{2}, \\
-\left(D_{1} \sqrt{M}\right)^{x_{2}^{\prime}-x_{1}^{\prime}} \sum_{m=0}^{\infty} \psi_{1}\left(x_{1}^{\prime}+m+1, \tau_{1}\right) \psi_{2}\left(x_{2}^{\prime}+m+1, \tau_{2}\right), & \tau_{1}<\tau_{2},\end{cases}
\end{aligned}
$$

where

$$
\begin{aligned}
& \psi_{1}\left(x_{1}^{\prime}, \tau_{1}\right)=\frac{1}{2 \pi i} \int_{-i \infty+\epsilon}^{i \infty+\epsilon} d z e^{\frac{z^{2}}{2}-\tau_{1} z} z^{x_{1}^{\prime}-1}, \\
& \psi_{2}\left(x_{2}^{\prime}, \tau_{2}\right)=\frac{1}{2 \pi i} \int_{C_{1}} \frac{d w}{w^{x_{2}^{\prime}+1}} e^{-\frac{w^{2}}{2}+\tau_{2} w} .
\end{aligned}
$$

Introducing the function $D_{n}(z)$ by

$$
D_{x-1}(\tau)=\sqrt{2 \pi} e^{\frac{\tau^{2}}{4}} \psi_{1}(x, \tau),
$$

one finds that the function satisfies Weber's equation [48],

$$
\frac{d^{2} D_{n}(z)}{d z^{2}}+\left(n+\frac{1}{2}-\frac{1}{4} z^{2}\right) D_{n}(z)=0 .
$$


The initial condition was given in [30] as

$$
\begin{aligned}
& D_{n}(0)=\frac{2^{\frac{n+1}{2}}}{\sqrt{2 \pi}} \sin \left(\frac{\pi}{2}(n+1)\right) \Gamma\left(\frac{n+1}{2}\right), \\
& D_{n}^{\prime}(0)=-D_{n+1}(0),
\end{aligned}
$$

where $\Gamma(x)$ represents the Gamma function. The function satisfying (5.21)-(5.23) is known as the parabolic cylinder function [48]. In addition, one easily finds $\psi_{2}(x, \tau)$ can be represented as (3.18) from the integral representation of the Hermite polynomial $H_{n}(x)$ [48],

$$
H_{n}(x)=\frac{n !}{2 \pi i} \oint \frac{d z}{z^{n+1}} e^{2 x z-z^{2}}
$$

Hence, noticing the prefactor $\left(D_{1} \sqrt{M}\right)^{x_{2}^{\prime}-x_{1}^{\prime}}$ does not contribute to the determinant in (3.3), we get the limiting kernel (3.16)-(3.18).

\subsection{Regions 2 and 3 (proof of Theorems 2-2 and 2-3')}

In this section we consider the scaling limit of the regions 2 and 3 in Fig. 3 . We fix the scaled time $u(3.8)$ such that $1 /(1-q)<u<u_{c}$ for the region 2 and $u=u_{c}$ for the region 3 , where $u_{c}=\left(\bar{q}^{2}-2 q \bar{q}+q\right) /(\bar{q}-q)^{2}$. We scale the time $t_{j}$, the position $\ell_{j}$ of the tagged particle at $t_{j}$, and the stay rates of the defect particles $\bar{q}_{i}(i=1, \cdots, n)$ as $(3.21),(3.22)$ and (3.36) respectively.

First we analyze the asymptotics of $\tilde{K}\left(t_{1}, x_{1} ; t_{2}, x_{2}\right)$ in $(3.6)$ by the saddle point method. Using the variable $\mu(u)$ which will be fixed later, we set

$$
\begin{aligned}
\tilde{K}\left(t_{1}, x_{1} ; t_{2}, x_{2}\right) & =\frac{1}{(2 \pi i)^{2}} \int_{C_{R_{1}}} \frac{d z_{1}}{z_{1}} \int_{C_{R_{2}}} \frac{d z_{2}}{z_{2}} \exp \left(M\left(f_{u_{2}}\left(z_{2}\right)-f_{u_{1}}\left(z_{1}\right)\right)\right) \frac{z_{1}}{z_{1}-z_{2}} \\
& \times \frac{z_{2}^{x_{2}-\mu\left(u_{2}\right) M}}{z_{1}^{x_{1}-\mu\left(u_{1}\right) M}}\left(\frac{1-p z_{1}}{1-p z_{2}}\right)^{n} \prod_{i=1}^{n} \frac{1-\bar{p}_{i} z_{2}}{1-\bar{p}_{i} z_{1}} \frac{1+1 / z_{2}}{1+1 / z_{1}} .
\end{aligned}
$$

Here $u_{i}=t_{i} / M, p=q /(1-q)$, and $\bar{p}_{i}=\bar{q}_{i} /\left(1-\bar{q}_{i}\right)$. The function $f_{u}(z)$ is defined as

$$
f_{u}(z)=(u-1) \log (1+z)+\log (1-p z)+(\mu(u)-u+1) \log (z) .
$$

We fix the value of $\mu(u)$ in such a way that two saddle points of $f_{u}(z)$ merge to one point. We have

$$
\mu(u)=u-1-A_{2}(u)=\frac{p(u-2)+2 \sqrt{p(u-1)}}{1+p},
$$

and the double critical point $z_{c}(u)$ of $f_{u}(z)$ is obtained as

$$
z_{c}(u)=\frac{\sqrt{u-1}-\sqrt{p}}{\sqrt{p^{2}(u-1)}+\sqrt{p}} .
$$


Note that

$$
f_{u}^{\prime}\left(z_{c}(u)\right)=f_{u}^{\prime \prime}\left(z_{c}(u)\right)=0
$$

is satisfied with $\mu(u)$ fixed as (5.27).

Thanks to the property that $z_{c}(u) \leq 1 / \bar{p}_{i}$ when $1 /(1-q)<u \leq u_{c}$ which is the condition of regions 2 and 3 , it is found that we can deform the contours $C_{R_{i}}(i=1,2)$ around the double saddle point (5.28). Thus we scale $z_{1}$ and $z_{2}$ as

$$
z_{1}=z_{c}\left(u_{1}\right)\left(1-\frac{i w_{1}}{D(u) M^{\frac{1}{3}}}\right), z_{2}=z_{c}\left(u_{2}\right)\left(1+\frac{i w_{2}}{D(u) M^{\frac{1}{3}}}\right),
$$

where $D(u)$ is defined in (3.24). Noting (5.29) and the relation

$$
-\frac{f^{\prime \prime \prime}\left(z_{c}(u)\right) z_{c}^{3}(u)}{2}=D(u)^{3},
$$

we have

$$
\begin{aligned}
\exp \left(M\left(f_{u_{2}}\left(z_{2}\right)-f_{u_{1}}\left(z_{1}\right)\right)\right) & \sim \frac{\exp \left(M f_{u_{2}}\left(z_{c}\left(u_{2}\right)\right)+M \frac{f_{u_{2}}^{\prime \prime \prime}\left(z_{c}\left(u_{2}\right)\right)}{6}\left(z_{2}-z_{c}\left(u_{2}\right)\right)^{3}\right)}{\exp \left(M f_{u_{1}}\left(z_{c}\left(u_{1}\right)\right)+M \frac{f_{u_{1}}^{\prime \prime \prime}\left(z_{c}\left(u_{1}\right)\right)}{6}\left(z_{1}-z_{c}\left(u_{1}\right)\right)^{3}\right)} \\
& \sim \exp \left(M\left(f_{u_{2}}\left(z_{c}\left(u_{2}\right)\right)-f_{u_{1}}\left(z_{c}\left(u_{1}\right)\right)\right)+\frac{i\left(w_{1}^{3}+w_{2}^{3}\right)}{3}\right) .
\end{aligned}
$$

Hence we had the asymptotic form of the term $\exp \left(M\left(f_{u_{2}}\left(z_{2}\right)-f_{u_{1}}\left(z_{1}\right)\right)\right)$ in (5.25). Next we consider that of other terms in (5.25). Combining (5.30) with the relation

$$
C(u) \frac{z_{c}^{\prime}(u)}{z_{c}(u)}=\frac{1}{D(u) M^{\frac{1}{3}}},
$$

we find

$$
z_{1} \sim z_{c}(u)\left(1+\frac{\tau_{1}-i w_{1}}{D(u) M^{\frac{1}{3}}}\right), z_{2} \sim z_{c}(u)\left(1+\frac{\tau_{2}+i w_{2}}{D(u) M^{\frac{1}{3}}}\right) .
$$

Furthermore, associated with (3.22), we scale $x_{i}(i=1,2)$ as

$$
x_{i}=(u-1) M-A_{2}\left(u_{i}\right) M+D(u) M^{\frac{1}{3}} \xi_{i} .
$$

Here $A_{2}(u)$ is defined in (3.12). Using (5.34), (5.35) and (3.36), we get

$$
\begin{aligned}
& \frac{z_{1}}{z_{1}-z_{2}} \sim \frac{D(u) M^{\frac{1}{3}}}{\tau_{1}-\tau_{2}-i\left(w_{1}+w_{2}\right)}, \\
& \left(\frac{1-p z_{1}}{1-p z_{2}}\right)^{n} \frac{1+1 / z_{2}}{1+1 / z_{1}} \sim 1, \\
& \prod_{i=1}^{n} \frac{1-\bar{p}_{i} z_{2}}{1-\bar{p}_{i} z_{1}} \sim \begin{cases}1, & \text { region } 2\left(u \leq u_{c}=\frac{\bar{q}^{2}-2 q \bar{q}+q}{(\bar{q}-q)^{2}}\right), \\
\prod_{i=1}^{n} \frac{\eta_{i}-\tau_{2}-i w_{2}}{\eta_{i}-\tau_{1}+i w_{1}}, & \text { region } 3\left(u=u_{c}\right),\end{cases} \\
& \frac{z_{2}^{x_{2}-\mu\left(u_{2}\right) M}}{z_{1}^{x_{1}-\mu\left(u_{1}\right) M} \sim z_{c}(u)^{D(u) M^{\frac{1}{3}}\left(\xi_{2}-\xi_{1}\right)} \exp \left(\xi_{2} \tau_{2}-\xi_{1} \tau_{1}+i w_{1} \xi_{1}+i w_{2} \xi_{2}\right) .}
\end{aligned}
$$


Note that in (5.38), the asymptotic form is different between regions 2 and 3. This leads to the difference of the limiting distribution between Theorems 2-2 and 2-3'.

Thus we obtain the asymptotic form of $\tilde{K}\left(t_{1}, x_{1} ; t_{2}, x_{2}\right)$,

$$
\begin{aligned}
& \tilde{K}\left(t_{1}, x_{1} ; t_{2}, x_{2}\right) \\
& \begin{cases}\sim \frac{\nu}{D(u) M^{\frac{1}{3}}} \int_{0}^{\infty} d \lambda e^{-\lambda\left(\tau_{1}-\tau_{2}\right)} \operatorname{Ai}\left(\xi_{1}+\lambda\right) \operatorname{Ai}\left(\xi_{2}+\lambda\right), & \text { region } 2, \\
\sim \frac{\nu}{D(u) M^{\frac{1}{3}}} \int_{0}^{\infty} d \lambda e^{-\lambda\left(\tau_{1}-\tau_{2}\right)} \operatorname{Ai}\left(\xi_{1}+\lambda\right) \operatorname{Ai}\left(\xi_{2}+\lambda\right) & \\
+\frac{\nu}{D(u) M^{\frac{1}{3}}} \sum_{j=1}^{n} \frac{1}{2 \pi} \int_{-\infty}^{\infty} d w_{1} \exp \left(i \xi_{1} w_{1}+\frac{i w_{1}^{3}}{3}\right) \prod_{k=1}^{j} \frac{1}{\eta_{k}-\tau_{1}+i w_{1}} & \\
\times \frac{1}{2 \pi} \int_{-\infty}^{\infty} d w_{2} \exp \left(i \xi_{2} w_{2}+\frac{i w_{2}^{3}}{3}\right) \prod_{k=1}^{j-1}\left(\eta_{k}-\tau_{2}+i w_{2}\right), & \text { region } 3\end{cases}
\end{aligned}
$$

Here

$$
\nu=\exp \left(M\left(f_{u_{2}}\left(z_{c}\left(u_{2}\right)\right)-f_{u_{1}}\left(z_{c}\left(u_{1}\right)\right)\right)+\xi_{2} \tau_{2}-\xi_{1} \tau_{1}\right) z_{c}(u)^{D(u) M^{\frac{1}{3}}\left(\xi_{2}-\xi_{1}\right)} .
$$

In (5.40), we used the integral representation of the Airy function

$$
\operatorname{Ai}(x)=\frac{1}{2 \pi} \int_{-\infty}^{\infty} d \lambda e^{i x \lambda+\frac{i}{3} \lambda^{3}}
$$

and the relation

$$
\begin{aligned}
& \frac{1}{\tau_{1}-\tau_{2}-i\left(w_{1}+w_{2}\right)}\left(\prod_{j=1}^{N} \frac{\eta_{j}-\tau_{2}-i w_{2}}{\eta_{j}-\tau_{1}+i w_{1}}-1\right) \\
& =\frac{1}{\eta_{1}-\tau_{1}+i w_{1}}+\frac{\eta_{1}-\tau_{2}-i w_{2}}{\left(\eta_{1}-\tau_{1}+i w_{1}\right)\left(\eta_{2}-\tau_{1}+i w_{1}\right)}+\cdots \\
& +\frac{\left(\eta_{1}-\tau_{2}-i w_{2}\right)\left(\eta_{2}-\tau_{2}-i w_{2}\right) \cdots\left(\eta_{N-1}-\tau_{2}-i w_{2}\right)}{\left(\eta_{1}-\tau_{1}+i w_{1}\right)\left(\eta_{2}-\tau_{1}+i w_{1}\right) \cdots\left(\eta_{N}-\tau_{1}+i w_{1}\right)} .
\end{aligned}
$$

Next we consider the asymptotics of $\phi_{t_{1}, t_{2}}\left(x_{1}, x_{2}\right)(3.7)$. Using $f_{u}(z)(5.26)$ and $\mu(u)(5.27)$, the function is rewritten as for $t_{1}<t_{2}$,

$$
\phi_{t_{1}, t_{2}}\left(x_{1}, x_{2}\right)=\frac{1}{2 \pi i} \int_{C_{1}} \frac{d z}{z} e^{M\left(f_{u_{2}}(z)-f_{u_{1}}(z)\right)} z^{x_{2}-\mu\left(u_{2}\right) M-x_{1}+\mu\left(u_{1}\right) M} .
$$

Let the variable $z$ scale as

$$
z=z_{c}(u)\left(1+\frac{i \sigma}{D(u) M^{\frac{1}{3}}}\right)
$$

where $z_{c}(u)$ defined in (5.28) is the double saddle point of (5.26). Due to (5.33), we have for $i=1,2$

$$
z \sim z_{c}\left(u_{i}\right)\left(1+\frac{i \sigma-\tau_{i}}{D(u) M^{\frac{1}{3}}}\right)
$$


From the above two equations, (5.31) and (5.35), we get

$$
\begin{aligned}
& e^{M\left(f_{u_{2}}(z)-f_{u_{1}}(z)\right)} \sim e^{M\left(f_{u_{2}}\left(z_{c}\left(u_{2}\right)\right)-f_{u_{1}}\left(z_{c}\left(u_{1}\right)\right)\right)} e^{\frac{i}{3}\left(\sigma+i \tau_{2}\right)^{3}-\frac{i}{3}\left(\sigma+i \tau_{1}\right)^{3}} \\
& =e^{M\left(f_{u_{2}}\left(z_{c}\left(u_{2}\right)\right)-f_{u_{1}}\left(z_{c}\left(u_{1}\right)\right)\right)} e^{-\left(\tau_{2}-\tau_{1}\right) \sigma^{2}-i\left(\tau_{2}^{2}-\tau_{1}^{2}\right) \sigma+\frac{\tau_{2}^{3}-\tau_{1}^{3}}{3}} \\
& z^{x_{2}-\mu\left(u_{2}\right) M-x_{1}+\mu\left(u_{1}\right) M} \sim z_{c}(u)^{D(u) M^{\frac{1}{3}}\left(\xi_{2}-\xi_{1}\right)} e^{i \sigma\left(\xi_{2}-\xi_{1}\right)} .
\end{aligned}
$$

Thus we obtain for $t_{1}<t_{2}$,

$$
\begin{aligned}
& \phi_{t_{1}, t_{2}}\left(x_{1}, x_{2}\right) \\
& \sim \frac{\nu}{D(u) M^{\frac{1}{3}}} e^{\xi_{1} \tau_{1}-\xi_{2} \tau_{2}+\frac{\tau_{2}^{3}-\tau_{1}^{3}}{3}} \int_{-\infty}^{\infty} d \sigma e^{-\left(\tau_{2}-\tau_{1}\right) \sigma^{2}-i\left(\tau_{2}^{2}-\tau_{1}^{2}\right) \sigma+i\left(\xi_{2}-\xi_{1}\right) \sigma} \\
& =\frac{\nu}{D(u) M^{\frac{1}{3}}} \frac{1}{\sqrt{4 \pi\left(\tau_{2}-\tau_{1}\right)}} \exp \left(\frac{-\left(\xi_{2}-\xi_{1}\right)^{2}}{4\left(\tau_{2}-\tau_{1}\right)}-\frac{\left(\xi_{2}+\xi_{1}\right)\left(\tau_{2}-\tau_{1}\right)}{2}+\frac{\left(\tau_{2}-\tau_{1}\right)^{3}}{12}\right),
\end{aligned}
$$

where $\nu$ is given in (5.41). From (5.42) we finally find

$$
\phi_{t_{1}, t_{2}}\left(x_{1}, x_{2}\right) \sim \frac{\nu}{D(u) M^{\frac{1}{3}}} \int_{-\infty}^{\infty} d \lambda e^{-\lambda\left(\tau_{1}-\tau_{2}\right)} \operatorname{Ai}\left(\xi_{1}+\lambda\right) \operatorname{Ai}\left(\xi_{2}+\lambda\right) .
$$

Hence from (5.43) and (5.50) and noting that the term $\nu$ (5.41) does not affect the determinant, we finally obtain the desired kernels for the regions 2 and 3.

\subsection{Region 4 (proof of Theorem 2-4')}

In this section, we discuss the asymptotics of the region 4 in Fig. 3. In this region we take time $t_{j}$ in such a way that they are macroscopically separated and the scaled time $u_{j}=t_{j} / M$ is taken as $u_{j}>u_{c}$. The position $\ell_{j}$ of the tagged particle at $t_{j}$ and the stay rates $\bar{q}_{i}(i=1, \cdots, n)$ of the defect particles are also scaled as (3.42) and (3.48) respectively.

Deforming the contour $C_{R_{1}}$ in the kernel $\tilde{K}\left(t_{1}, x_{1} ; t_{2}, x_{2}\right)$ (3.6), we divide it into two parts,

$$
\tilde{K}\left(t_{1}, x_{1} ; t_{2}, x_{2}\right)=\tilde{K}_{1}\left(t_{1}, x_{1} ; t_{2}, x_{2}\right)-\tilde{K}_{2}\left(t_{1}, x_{1} ; t_{2}, x_{2}\right) .
$$

Here for $i=1,2$

$$
\begin{aligned}
& \tilde{K}_{i}\left(t_{1}, x_{1} ; t_{2}, x_{2}\right) \\
& =-\frac{1}{(2 \pi i)^{2}} \int_{\Gamma_{\bar{p}}^{(i)}} \frac{d z_{1}}{z_{1}} \int_{C_{R_{2}}} \frac{d z_{2}}{z_{2}} \frac{z_{1}}{z_{1}-z_{2}} \frac{\left(1+1 / z_{2}\right)^{t_{2}-M+1}}{\left(1+1 / z_{1}\right)^{t_{1}-M+1}}\left(\frac{1-p z_{2}}{1-p z_{1}}\right)^{M-n} \prod_{i=1}^{n} \frac{1-\bar{p}_{i} z_{2}}{1-\bar{p}_{i} z_{1}} \frac{z_{2}^{x_{2}}}{z_{1}^{x_{1}}},
\end{aligned}
$$

where $p=q /(1-q)$ and $\bar{p}_{i}=\bar{q}_{i} /\left(1-\bar{q}_{i}\right)$. In the equation above, the contour $\Gamma_{\bar{p}}^{(1)}$ encloses $z=1 / \bar{p}_{i}$ anticlockwise while the contour $\Gamma_{\bar{p}}^{(2)}$ is chosen in such a way that it encloses $z=-1,0,1 / \bar{p}_{i}$ anticlockwise. 
First, we discuss the asymptotic form of $\tilde{K}_{1}\left(t_{1}, x_{1} ; t_{2}, x_{2}\right)$. This is rewritten as

$$
\begin{aligned}
\tilde{K}_{1}\left(t_{1}, x_{1} ; t_{2}, x_{2}\right)=-\frac{1}{(2 \pi i)^{2}} \int_{\Gamma_{\bar{p}}^{(1)}} \frac{d z_{1}}{z_{1}} \int_{C_{R_{2}}} \frac{d z_{2}}{z_{2}} e^{M\left(g_{u_{2}}\left(z_{2}\right)-g_{u_{1}}\left(z_{1}\right)\right)} \frac{z_{1}}{z_{1}-z_{2}} \\
\times\left(\frac{1-p z_{1}}{1-p z_{2}}\right)^{n} \prod_{i=1}^{n} \frac{1-\bar{p}_{i} z_{2}}{1-\bar{p}_{i} z_{1}} \frac{1+1 / z_{2}}{1+1 / z_{1}} \frac{z_{2}^{x_{2}-M \Lambda\left(u_{2}\right)}}{z_{1}^{x_{1}-M \Lambda\left(u_{1}\right)}},
\end{aligned}
$$

where $\Lambda(u)$ is a variable which will be fixed later and

$$
g_{u}(z)=(u-1) \log (1+z)+\log (1-p z)+(\Lambda(u)-u+1) \log z .
$$

We choose the value of $\Lambda(u)$ in such a way that the saddle point of $g_{u}(z)$ is $1 / \bar{p}$ where $\bar{p}=\bar{q} /(1-\bar{q})$. From the condition,

$$
g_{u}^{\prime}\left(\frac{1}{\bar{p}}\right)=0
$$

we have

$$
\Lambda(u)=(u-1)-A_{G}(u)=\frac{\bar{p}(u-1)}{1+\bar{p}}+\frac{p}{\bar{p}-p} .
$$

Scaling $z_{i}(i=1,2)$ around the saddle point (5.55) as

$$
z_{i}=\frac{1}{\bar{p}}\left(1-\frac{2 w_{i}}{D_{G}\left(u_{i}\right) M^{\frac{1}{2}}}\right)
$$

and noting $\bar{p}^{2} D_{G}^{2}(u)=2 g_{u}^{\prime \prime}(1 / \bar{p})$, we can obtain the asymptotic form of $e^{M\left(g_{u_{2}}\left(z_{2}\right)-g_{u_{1}}\left(z_{1}\right)\right)}$ in (5.53) by the saddle point method,

$$
e^{M\left(g_{u_{2}}\left(z_{2}\right)-g_{u_{1}}\left(z_{1}\right)\right)} \sim e^{M\left(g_{u_{2}}\left(\frac{1}{p}\right)-g_{u_{1}}\left(\frac{1}{\bar{p}}\right)\right)} e^{z_{2}^{2}-z_{1}^{2}} .
$$

Associated with (3.42), we set

$$
x_{i}=\left(u_{i}-1\right) M-A_{G}\left(u_{i}\right) M+D_{G}\left(u_{i}\right) M^{\frac{1}{2}} \xi_{i},
$$

for $i=1,2$. From this equation, (5.57) and (3.48), we find

$$
\begin{aligned}
& \prod_{i=1}^{n} \frac{1-\bar{p}_{i} w}{1-\bar{p}_{i} z} \sim \prod_{i=1}^{n} \frac{e^{-\tau_{2}} w_{2}+\epsilon_{i}}{e^{-\tau_{1}} w_{1}+\epsilon_{i}}, \frac{z_{1}}{z_{1}-z_{2}} \sim \frac{M^{\frac{1}{2}}}{-2\left(e^{-\tau_{1}} w_{1}-e^{-\tau_{2}} w_{2}\right)} \\
& \left(\frac{1-p z_{1}}{1-p z_{2}}\right)^{n} \frac{1+1 / z_{2}}{1+1 / z_{1}} \sim 1, \quad \frac{z_{2}^{x_{2}-M \Lambda\left(u_{2}\right)}}{z_{1}^{x_{1}-M \Lambda\left(u_{1}\right)}} \sim\left(\frac{1}{\bar{p}}\right)^{\left(e^{\left.\tau_{2} \xi_{2}-e^{\tau_{1}} \xi_{1}\right) M^{\frac{1}{2}}} e^{-2 w_{2} \xi_{2}+2 w_{1} \xi_{1}}\right.}
\end{aligned}
$$

where we used the parameter $\tau_{i}$ defined in (3.44). 
Thus we eventually get

$$
\begin{aligned}
& \tilde{K}_{1}\left(t_{1}, x_{1} ; t_{2}, x_{2}\right) \\
& \sim \frac{(1+\bar{p})^{t_{2}-t_{1}} \bar{p}^{x_{1}-x_{2}}}{e^{\tau_{2}} M^{\frac{1}{2}}} \frac{2}{(2 \pi i)^{2}} \int_{\Gamma} d w_{1} \int_{\gamma} d w_{2} \frac{e^{w_{2}^{2}-w_{1}^{2}-2 w_{2} \xi_{2}+2 w_{1} \xi_{1}}}{e^{\tau_{1}-\tau_{2}} w_{2}-w_{1}} \prod_{i=1}^{n} \frac{e^{-\tau_{2}} w_{2}+\epsilon_{i}}{e^{-\tau_{1}} w_{1}+\epsilon_{i}}
\end{aligned}
$$

Here the contour $\Gamma$ encloses $-e^{\tau_{1}} \epsilon_{i}(i=1, \cdots, n)$ anticlockwise and $\gamma$ is an arbitrary path running from $-i \infty$ to $i \infty$.

Next we consider the asymptotics of $\phi_{t_{1}, t_{2}}\left(x_{1}, x_{2}\right)$ (3.7). By use of $g_{u}(z)$ (5.54) and $\Lambda(u)(5.56)$, we set for $t_{1}<t_{2}$,

$$
\phi_{t_{1}, t_{2}}\left(x_{1}, x_{2}\right)=\frac{1}{2 \pi i} \int_{C_{1}} \frac{d z}{z} e^{M\left(g_{u_{2}}(z)-g_{u_{1}}(z)\right)} z^{x_{2}-x_{1}-\Lambda\left(u_{2}\right)+\Lambda\left(u_{1}\right)} .
$$

Scaling $z$ as

$$
z=\frac{1}{\bar{p}}\left(1+i \frac{2 w}{\sqrt{\left(D_{G}^{2}\left(u_{2}\right)-D_{G}^{2}\left(u_{1}\right)\right) M}}\right),
$$

From this equation and (5.59), we get

$$
\begin{aligned}
& e^{M\left(g_{u_{2}}(z)-g_{u_{1}}(z)\right)} \sim e^{M\left(g_{u_{2}}\left(\frac{1}{\bar{p}}\right)-g_{u_{1}}\left(\frac{1}{\bar{p}}\right)\right)} e^{-w^{2}} \\
& z^{x_{2}-x_{1}-M\left(\Lambda\left(u_{2}\right)-\Lambda\left(u_{1}\right)\right)} \sim\left(\frac{1}{\bar{p}}\right)^{\left(e^{\tau_{2}} \xi_{2}-e^{\tau_{1}} \xi_{1}\right) M^{\frac{1}{2}}} \exp \left(2 i w \frac{\xi_{2}-e^{\tau_{1}-\tau_{2}} \xi_{1}}{\sqrt{1-e^{2\left(\tau_{1}-\tau_{2}\right)}}}\right)
\end{aligned}
$$

Thus we obtain for $t_{1}<t_{2}$,

$$
\begin{aligned}
\phi_{t_{1}, t_{2}}\left(x_{1}, x_{2}\right) & \sim \frac{(1+\bar{p})^{t_{2}-t_{1}} \bar{p}^{x_{1}-x_{2}}}{\pi \sqrt{\left(e^{2 \tau_{2}}-e^{2 \tau_{1}}\right) M}} \int_{-\infty}^{\infty} d w e^{-w^{2}+2 i w \frac{\xi_{2}-e^{\tau_{1}-\tau_{2} \xi_{1}}}{\sqrt{1-e^{2\left(\tau_{1}-\tau_{2}\right)}}}} \\
& =\frac{(1+\bar{p})^{t_{2}-t_{1}} \bar{p}^{x_{1}-x_{2}}}{e^{\tau_{2}} M^{\frac{1}{2}}} \frac{e^{-\frac{\left(\xi_{2}-e^{\tau_{1}-\tau_{2}} \xi_{1}\right)^{2}}{1-e^{2\left(\tau_{1}-\tau_{2}\right)}}}}{\sqrt{\pi\left(1-e^{2\left(\tau_{1}-\tau_{2}\right)}\right)}}
\end{aligned}
$$

At last, we discuss that $\tilde{K}_{2}\left(t_{1}, x_{1} ; t_{2}, x_{2}\right)$ in (5.52) does not contribute the asymptotic form of the kernel (3.5). The derivation can be done in the similar fashion to the one in Theorem 3.1. in [54]. Here we give only its outline.

First we consider the case of equal time, $t_{1}=t_{2}=t=u M$. Scaling $z_{i}(i=1,2)$ as (5.30), we have

$$
\begin{aligned}
& \tilde{K}_{2}\left(t, x_{1} ; t, x_{2}\right) \sim \frac{-z_{c}(u)^{D_{G}(u) M^{\frac{1}{2}}\left(\xi_{2}-\xi_{1}\right)}}{D(u) M^{\frac{1}{3}}} \\
& \times \int_{0}^{\infty} d \lambda \operatorname{Ai}\left(\frac{\Delta A(u)}{D(u)} M^{\frac{2}{3}}+\frac{D_{G}(u)}{D(u)} M^{\frac{1}{6}} \xi_{1}+\lambda\right) \mathrm{Ai}\left(\frac{\Delta A(u)}{D(u)} M^{\frac{2}{3}}+\frac{D_{G}(u)}{D(u)} M^{\frac{1}{6}} \xi_{2}+\lambda\right),
\end{aligned}
$$


where $\Delta A(u)=A_{2}(u)-A_{G}(u)$. Noticing that $\Delta A(u)>0$ for the region $4\left(u_{c}<u\right)$ and the asymptotic form of the Airy function

$$
\operatorname{Ai}(x) \sim \frac{1}{2 \sqrt{\pi}} x^{-\frac{1}{4}} \exp \left(-\frac{2}{3} x^{\frac{3}{2}}\right)
$$

as $x \rightarrow \infty$, we find

$$
\tilde{K}_{2}\left(t, x_{1} ; t, x_{2}\right) \sim e^{-\mathcal{O}(M)} .
$$

This indicates that under the scaling (3.42), the kernel $\tilde{K}_{2}\left(t, x_{1} ; t, x_{2}\right)$ vanishes as $M$ goes to infinity.

Next we consider the case for arbitrary $t_{i}(i=1,2)$. We scale $z_{1}$ around the double saddle point $z_{c}\left(u_{1}\right)$ of $f_{u_{1}}(z)$ and $z_{2}$ around the saddle point $1 / \bar{p}$ of $g_{u_{2}}\left(z_{1}\right)$ as

$$
z_{1}=z_{c}\left(u_{1}\right)\left(1-\frac{i w_{1}}{D\left(u_{1}\right) M^{\frac{1}{3}}}\right), z_{2}=\frac{1}{\bar{p}}\left(1-\frac{2 w_{2}}{D_{G}\left(u_{2}\right) M^{\frac{1}{2}}}\right) .
$$

Under these scalings, we find

$$
\tilde{K}_{2}\left(t, x_{1} ; t, x_{2}\right) \sim-\frac{(1+\bar{p})^{t_{2}-t_{1}} \bar{p}^{x_{1}-x_{2}} e^{-\xi_{2}^{2}} D_{G}\left(u_{1}\right)}{\sqrt{\pi} D_{G}\left(u_{2}\right)} \times \Theta\left(u_{1}, \xi_{1}\right),
$$

where

$$
\begin{aligned}
& \Theta\left(u_{1}, \xi_{1}\right) \\
= & \frac{1}{z_{c}\left(u_{1}\right)-1 / \bar{p}}\left(\frac{1-p / \bar{p}}{1-p z_{c}\left(u_{1}\right)}\right)^{M-n} \prod_{i=1}^{n} \frac{1-\bar{p}_{i} / \bar{p}}{1-\bar{p}_{i} z_{c}\left(u_{1}\right)} \frac{z_{c}\left(u_{1}\right)^{-x_{1}} \bar{p}^{-x_{1}}}{D\left(u_{1}\right) D_{G}\left(u_{1}\right) M^{\frac{5}{6}} \bar{p}} \\
& \times \frac{(1+\bar{p})^{\left(u_{1}-1\right) M+1}}{\left(1+\frac{1}{z_{c}\left(u_{1}\right)}\right)^{\left(u_{1}-1\right) M+1}} \operatorname{Ai}\left(\frac{\Delta A\left(u_{1}\right)}{D\left(u_{1}\right)} M^{\frac{2}{3}}+\frac{D_{G}\left(u_{1}\right)}{D\left(u_{1}\right)} M^{\frac{1}{6}} \xi_{1}\right) .
\end{aligned}
$$

Considering the case $t_{1}=t_{2}$ in (5.71) and the former result (5.69), one easily finds

$$
\Theta(u, \xi) \sim e^{-\mathcal{O}(M)}
$$

Hence we finally get

$$
\tilde{K}_{2}\left(t_{1}, x_{1} ; t_{2}, x_{2}\right) \sim(1+\bar{p})^{t_{2}-t_{1}} \bar{p}^{x_{1}-x_{2}}\left(e^{-\mathcal{O}(M)}\right) .
$$

From $(5.61),(5.66)$ and $(5.74)$ and noting the factor $(1+\bar{p})^{t_{2}-t_{1}} \bar{p}^{x_{1}-x_{2}}$ does not affect the determinant, we obtain the desired expression for the limiting kernel. 


\subsection{Fixed $M$ case (proof of Theorem 3)}

In this section we discuss the scaling limit explained in Section 3.3 where time $t$ goes to infinity with $M$ fixed. In this limit, we scale the time $t_{j}$, the particle position $\ell_{j}$, and the stay rates $q_{i}(i=1, \cdots, M)$ as $(3.54),(3.55)$, and (3.56) respectively.

First we consider the scaling form of $\tilde{K}\left(t_{1}, x_{1} ; t_{2}, x_{2}\right)$ (3.6). Changing the variables $z_{i}(i=1,2)$ to $z_{i}^{\prime}=1 / z_{i}$, we have

$$
\tilde{K}\left(t_{1}, x_{1} ; t_{2}, x_{2}\right)=\frac{1}{(2 \pi i)^{2}} \int_{\Gamma_{p}} \frac{d z_{1}^{\prime}}{z_{1}^{\prime}} \int_{C_{R_{1}}} \frac{d z_{2}^{\prime}}{z_{2}^{\prime}} \frac{z_{2}^{\prime}}{z_{2}^{\prime}-z_{1}^{\prime}} \frac{\left(1+z_{2}^{\prime}\right)^{t_{2}-M+1}}{\left(1+z_{1}^{\prime}\right)^{t_{1}-M+1}} \prod_{i=1}^{M} \frac{z_{2}^{\prime}-p_{i}}{z_{1}^{\prime}-p_{i}} \frac{z_{1}^{\prime x_{1}+M}}{z_{2}^{\prime x_{2}+M}},
$$

where the contour $\Gamma_{p}$ encloses $p_{i}=q_{i} /\left(1-q_{i}\right)$ anticlockwise, and $C_{R_{1}}$ encloses the origin $z^{\prime}$ and $p_{i}=q_{i} /\left(1-q_{i}\right)$. Introducing $y\left(\tau_{i}\right)(i=1,2)$, which will be chosen later, we set

$$
\begin{aligned}
& \tilde{K}\left(t_{1}, x_{1} ; t_{2}, x_{2}\right) \\
& =\frac{1}{(2 \pi i)^{2}} \int_{\Gamma_{p}} \frac{d z_{1}^{\prime}}{z_{1}^{\prime}} \int_{C_{R_{1}}} \frac{d z_{2}^{\prime}}{z_{2}^{\prime}} \frac{z_{2}^{\prime}}{z_{2}^{\prime}-z_{1}^{\prime}} e^{T\left(h_{\tau_{2}}\left(z_{2}^{\prime}\right)-h_{\tau_{1}}\left(z_{1}^{\prime}\right)\right)} \prod_{i=1}^{M} \frac{z_{2}^{\prime}-p_{i}}{z_{1}^{\prime}-p_{i}} \frac{z_{1}^{\prime x_{1}-y\left(\tau_{1}\right) T+M}}{z_{2}^{\prime x_{2}-y\left(t_{2}\right) T+M}}\left(\frac{1+z_{1}^{\prime}}{1+z_{2}^{\prime}}\right)^{M-1},
\end{aligned}
$$

where the parameter $\tau$ is defined in (3.54) and

$$
h_{\tau}(z)=e^{2 \tau} \ln (1+z)-y(\tau) \ln (z) .
$$

We choose $y(\tau)$ in a way that $h_{\tau}(z)$ has the saddle point at $z=p=q /(1-q)$ where $q$ is defined in (3.56). Thus from the condition, $h_{\tau}^{\prime}(p)=0$, we have

$$
y(\tau)=\frac{p e^{2 \tau}}{1+p} .
$$

We scale the variable $z_{i}^{\prime}(i=1,2)$ around the saddle point $z_{c}=p$ as

$$
z_{i}^{\prime}=p\left(1+\frac{\sqrt{2}(1+p)}{e^{\tau_{i}}(p T)^{\frac{1}{2}}} w_{i}\right)
$$

Considering (3.55), we also scale $x_{i}(i=1,2)$ as

$$
x_{i}=\frac{p e^{2 \tau_{i}}}{1+p} T+\xi_{i} \frac{e^{\tau_{i}} \sqrt{2 p T}}{1+p} .
$$

From these equations and (3.56), we find

$$
\begin{array}{ll}
e^{T\left(h_{\tau_{2}}\left(z_{2}^{\prime}\right)-h_{\tau_{1}}\left(z_{1}^{\prime}\right)\right)} \sim e^{T\left(h_{\tau_{2}}(p)-h_{\tau_{1}}(p)\right)} e^{w_{2}^{2}-w_{1}^{2}}, & \frac{z_{2}^{\prime}}{z_{2}^{\prime}-z_{1}^{\prime}} \sim \frac{1}{e^{-\tau_{2}} w_{2}-e^{-\tau_{1}} w_{1}} \frac{(p T)^{\frac{1}{2}}}{\sqrt{2}(1+p)}, \\
\frac{z_{1}^{\prime x_{1}-y\left(\tau_{1}\right) T}}{z_{2}^{\prime x_{2}-y\left(\tau_{2}\right) T}} \sim p^{\frac{\sqrt{2 p T}}{1+p}\left(e^{\tau_{1}} \xi_{1}-e^{\tau_{2}} \xi_{2}\right)} e^{2 w_{1} \xi_{1}-2 w_{2} \xi_{2}}, & \prod_{i=1}^{M} \frac{z_{2}^{\prime}-p_{i}}{z_{1}^{\prime}-p_{i}} \sim \prod_{j=1}^{M} \frac{e^{-\tau_{2}} w_{2}+\epsilon_{j}}{e^{-\tau_{1}} w_{1}+\epsilon_{j}} \\
\frac{z_{1}^{\prime M}}{z_{2}^{\prime M}}\left(\frac{1+z_{1}^{\prime}}{1+z_{2}^{\prime}}\right)^{M-1} \sim 1 .
\end{array}
$$


From these equations, we eventually obtain

$$
\begin{aligned}
& \tilde{K}\left(t_{1}, x_{1} ; t_{2}, x_{2}\right) \\
& \sim(1+p)^{t_{2}-t_{1}} p^{x_{1}-x_{2}} \frac{(1+p)}{e^{\tau_{2}} \sqrt{2 p T}} \frac{2}{(2 \pi i)^{2}} \int_{\Gamma} d w_{1} \int_{\gamma} d w_{2} \frac{e^{w_{2}^{2}-2 w_{2} \xi_{2}-w_{1}^{2}+2 w_{1} \xi_{1}}}{e^{\tau_{1}-\tau_{2}} w_{2}-w_{1}} \prod_{j=1}^{M} \frac{e^{-\tau_{2}} w_{2}+\epsilon_{j}}{e^{-\tau_{1}} w_{1}+\epsilon_{j}} .
\end{aligned}
$$

Here the contour $\Gamma$ encloses $-e^{\tau_{1}} \epsilon_{i},(i=1, \cdots, M)$ anticlockwise and $\gamma$ is arbitrary path from $-i \infty$ to $i \infty$.

Next we consider the scaling limit of $\phi_{t_{1}, t_{2}}\left(x_{1}, x_{2}\right)(3.7)$. Changing the variable $z$ to $z^{\prime}=1 / z$, we set

$$
\phi_{t_{1}, t_{2}}\left(x_{1}, x_{2}\right)=\frac{1}{2 \pi i} \oint \frac{d z^{\prime}}{z^{\prime}} e^{T\left(h_{\tau_{2}}\left(z^{\prime}\right)-h_{\tau_{1}}\left(z^{\prime}\right)\right)} z^{x_{1}-x_{2}-\left(y\left(\tau_{1}\right)-y\left(\tau_{2}\right)\right) T},
$$

where $h_{\tau}(z)$ and $y(\tau)$ are given in (5.77) and (5.78). We set

$$
z^{\prime}=p\left(1+\sqrt{\frac{2}{\left(e^{2 \tau_{2}}-e^{2 \tau_{1}}\right) p T}}(1+p) w\right) .
$$

From this equation and (5.80)

$$
\begin{aligned}
& e^{T\left(h_{\tau_{2}}(z)-h_{\tau_{1}}(z)\right)} \sim e^{T\left(h_{\tau_{2}}(z)-h_{\tau_{1}}(z)\right)} e^{w^{2}} \\
& z^{x_{1}-x_{2}-\left(y_{\tau_{1}}-y_{\tau_{2}}\right) T} \sim p^{\left(\tau_{2} \xi_{2}-\tau_{1} \xi_{1}\right) \frac{\sqrt{2 p T}}{1+p}} \exp \left(\frac{2\left(\xi_{2}-\xi_{1}\right) w}{\sqrt{e^{2 \tau_{2}}-e^{2 \tau_{1}}}}\right) .
\end{aligned}
$$

Combining these equations, we finally find

$$
\begin{aligned}
& \phi_{t_{1}, t_{2}}\left(x_{1}, x_{2}\right) \\
& \sim(1+p)^{t_{2}-t_{1}} p^{x_{1}-x_{2}} \frac{\sqrt{2}(1+p)}{\sqrt{\left(e^{2 \tau_{2}}-e^{2 \tau_{1}}\right) T p}} \frac{1}{2 \pi i} \int_{-\infty}^{\infty} d w \exp \left(w^{2}+\frac{2\left(\xi_{2}-\xi_{1}\right) w}{\sqrt{e^{2 \tau_{2}}-e^{2 \tau_{1}}}}\right), \\
& =(1+p)^{t_{2}-t_{1}} p^{x_{1}-x_{2}} \frac{1+p}{e^{\tau_{2}} \sqrt{2 p T}} \sqrt{\frac{1}{\pi\left(1-e^{2\left(\tau_{2}-\tau_{1}\right)}\right)}} \exp \left(\frac{-\left(\xi_{2}-e^{\tau_{1}-\tau_{2}} \xi_{1}\right)^{2}}{1-e^{2\left(\tau_{1}-\tau_{2}\right)}}\right) .
\end{aligned}
$$

Thus from (5.82) and (5.86) and noting that the prefactor $(1+p)^{t_{2}-t_{1}} p^{x_{1}-x_{2}}$ does not affect the determinant, we get the desired expression for the kernel.

\section{Discussion}

\subsection{Numerical simulations}

In this section, we give the result of Monte-Carlo simulations about the position fluctuation of a tagged particle in the TASEP in order to check our analysis discussed in the preceding sections. 
We performed the simulations in two situations, the case without defect particle and that with one defect particle. Fig. 8 shows the data of the scaled position of a tagged particle obtained by the Monte-Carlo simulation and the probability distribution functions which must fit them. Fig. 8(a) corresponds to the time region $1 /(1-q)<u<u_{c}=$ $\left(\bar{q}^{2}-2 q \bar{q}+q\right) /(\bar{q}-q)^{2}$ where $q$ (resp. $\bar{q}$ ) is the stay rate of the normal particle (resp. the defect particle). Note that in the region, the both situations belong to the region 2 and due to Theorem 2-2, the GUE Tracy-Widom distribution must fit the data. Fig. 8(b) represents the case $u=u_{c}$. In this case the first situation comes under the region 2 . On the other hand, the second one belongs to the region 3 where the position fluctuation are described by the limiting largest eigenvalue distribution of $\mathrm{GOE}^{2}$ as explained in Theorem 2-3. In Fig. 8(c), only the data for the second situation are shown for the case $u_{c}<u$. This case is classified as the region 4 and from Theorem 2-4, the fluctuation is supposed to be Gaussian. In all figures, we see a good agreement between the data and the distribution functions which they must obey.

\subsection{Correlation of current fluctuations}

In this article, we have discussed the multi-time distribution of the tagged particle (2.1). In order to analyze it, we have introduced the directed polymer problem of the 01 matrix in Section 4.

Here we consider the directed polymer problem of another random matrix where each element is geometric random variable. By this analysis we can discuss the correlations of other quantities in the TASEP with the step initial condition.

Let $\left\{a_{g}(i, j)\right\}_{1 \leq i \leq N, 1 \leq j \leq M}$ be the $N \times M$ matrix and the element $a_{g}(i, j)$ is the geometric random variable,

$$
\operatorname{Prob}\left(a_{g}(i, j)=k\right)=\left(1-q_{j}\right) q_{j}^{k},
$$

where $q_{j}$ is the parameter of the geometric distribution and we identified this with the stay rate of the $j$ th particle of the TASEP with the step initial condition defined in Section 2 . For the matrix, we introduce the quantity $G_{g}^{*}(N, M)$ analogous to $G(N, M)(4.3)$ as

$$
G_{g}^{*}(N, M)=N+M-1+\max _{\pi_{g}(N, M)} \sum_{(i, j) \in \pi_{g}(N, M)} a_{g}(i, j) .
$$

Here $\pi_{g}(N, M)$ is the set of right/down paths from $(1,1)$ to $(N, M)$,

$$
\begin{aligned}
\pi_{g}(N, M)= & \left\{\left\{\left(i_{k}, j_{k}\right)_{k=1,2, \cdots, N+M-1}\right\} \mid\left(i_{1}, j_{1}\right)=(1,1),\left(i_{N+M-1}, j_{N+M-1}\right)=(N, M),\right. \\
& \left.\left(i_{k+1}-i_{k}, j_{k+1}-j_{k}\right)=(1,0) \text { or }(0,1)\right\} .
\end{aligned}
$$

In this setting, we consider the following quantity,

$$
\operatorname{Prob}\left(G_{g}^{*}\left(N_{1}, M\right) \leq t_{1}, \cdots, G_{g}^{*}\left(N_{m}, M\right) \leq t_{m}\right) .
$$

It is known that $G_{g}^{*}(N, M)$ can be interpreted as a quantity in the TASEP as follows [17, 43], 
$G_{g}^{*}(N, M)=t$ : In the TASEP with the step initial condition, the time until which the $M$ th particle moves $N$ sites to its right is $t$.

Thus the quantity (6.4) represents the correlations of the arrival times of the $M$ th particle at the site $N_{i}(i=1, \cdots, m)$. (Note that we set the site coordinate as in Fig. 1(b).) Furthermore we easily find that

$$
\operatorname{Prob}\left(G_{g}^{*}(N, M) \leq t\right)= \begin{cases}\operatorname{Prob}(H(t, N) \geq N), & \text { for } N \leq M \\ \operatorname{Prob}(H(t, N)=M), & \text { for } M \leq N\end{cases}
$$

Here $H(t, N)$ represents the number of particles which passed the site $N$ until time $t$. Thus the probability (6.4) also means the correlations of currents between different times and different sites.

Applying the similar technique in Section 4 to (6.4), we can also represent it as the growth process of Young diagram characterized by the Schur process. The result is as follows,

$$
\begin{aligned}
& \operatorname{Prob}\left(G_{g}^{*}\left(N_{1}, M\right) \leq t_{1}, \cdots, G_{g}^{*}\left(N_{m}, M\right) \leq t_{m}\right) \\
& =\operatorname{Prob}\left(\lambda_{1}\left(N_{1}, M\right) \leq t_{1}-N-M+1, \cdots, \lambda_{1}\left(N_{m}, M\right) \leq t_{m}-N-M+1\right),
\end{aligned}
$$

where $\lambda\left(N_{i}, M\right)$ is the Young diagram obtained by applying the normal RSK algorithm to the submatrix $\left\{a_{g}(i, j)\right\}_{i=1, \cdots, N_{i}, j=1, \cdots, M}$ and $\lambda_{1}\left(N_{i}, M\right)$ is the length of its first row. The probability measure in the right hand side of this equation is characterized by the following joint distribution function,

$$
\begin{aligned}
& \operatorname{Prob}\left(\lambda(1, M)=\lambda^{(1)}, \cdots, \lambda(N, M)=\lambda^{(N)}\right) \\
& =s_{\lambda^{(N)}}\left(q_{1}, q_{2}, \cdots, q_{M}\right) s_{\lambda^{(1)}}(1,0, \cdots) s_{\lambda^{(2)} / \lambda^{(1)}}(1,0, \cdots) \cdots s_{\lambda^{(N)} / \lambda^{(N-1)}}(1,0, \cdots) \prod_{i=1}^{M}\left(1-q_{i}\right)^{N},
\end{aligned}
$$

where $s_{\lambda / \mu}\left(q_{1}, q_{2}, \cdots\right)$ is the Schur function. This is also a special case of the Schur process and we can get the Fredholm determinant representation of (6.6). Although in one point case, the asymptotics of this equation was discussed in [17, 43], the multi-point distribution has not been discussed yet.

\section{Conclusion}

In this article, we have studied the multi-time distribution function (2.1) of position fluctuations of a tagged particle in the TASEP with the step initial condition. The main results are summarized as Theorems 1, 2 and 3 in Section 3.

First, we have obtained the Fredholm determinant expression of (2.1) in Theorem 1. For this purpose, we have mapped the time evolution of a tagged particle in the TASEP 
to the growth process of Young diagram which is related to the special case of the Schur process. Next, using the Fredholm determinant in Theorem 1, we have studied the two types of scaling limit. The first one is the case where both time $t$ and the label of a tagged particle $M$ go to infinity. In the second one, we take the $t \rightarrow \infty$ limit with $M$ fixed. The results for the first and second ones are shown in Theorems 2 and 3 respectively in Section 3.

In the first scaling limit, if the hopping rates of all particles are the same, we can divide the scaled time into two characteristic regions according to the limiting behavior of (2.1), the region 1 where a tagged particle begins to move and the region 2 which is after the region 1 . In the region 1 , the limiting process of a tagged particle converges to the spatially discrete process which is described in Theorem 2-1. The process reflects on the discreteness of the model. In the region 2, on the other hand, we have shown in Theorem 2-2 that the limiting process becomes the Airy process, which is characteristic of the one-dimensional KPZ universality class.

If there are $n$ defect particles with small hoping rates in front of a tagged particle, the limiting distribution changes at the scaled time $u_{c}$ which is determined by the hopping rate of the slowest defect particle. The limiting process around $u_{c}$ (region 3 ) is equivalent to that of the largest eigenvalue in GUE Dyson's Brownian motion with rank one external source as described in Theorem 2-3. Theorem 2-3' indicates that the rank becomes $n$ if the hopping rates of the defect particles are the same. When the scaled time is after $u_{c}$ (region 4 ), we have found in Theorem 2-4 that the process is equivalent to the one dimensional Brownian motion. If the hopping rates are degenerate, it is equivalent to the process of the largest eigenvalue of $n \times n$ GUE Dyson's Brownian motion model as described in Theorem 2-4'. This indicates that in the region 4, the effect of the defect particles is dominant whereas that of an infinite number of normal particles is irrelevant.

Theorem 3 shows the result for the second scaling limit. The limiting distribution is also described as the Fredholm determinant whose kernel is the same as that in Theorem $2-4$ '.

\section{Acknowledgments}

The authors would like to thank A. Borodin for drawing out attention to the reference [49]. The work of T.I. is supported by Core Research for Evolutional Science and Technology of Japan Science and Technology Agency. The work of T.S. is supported by the Grantin-Aid for Young Scientists (B), the Ministry of Education, Culture, Sports, Science and Technology, Japan.

\section{References}

[1] T. M. Liggett, Interacting Particle Systems, Splinger-Verlag, New York, 1985. 
[2] T. M. Liggett, Stochastic Interacting Systems: Contact, Voter, and Exclusion Processes, Splinger-Verlag, New York, 1999.

[3] H. Spohn, Large Scale Dynamics of Interacting Particles, Splinger-Verlag, New York, 1991.

[4] B. Schmittmann and R. K. P. Zia, Statistical mechanics of driven diffusive systems, in C. Domb and J. Lebowitz eds., Phase Transitions and Critical Phenomena, 17, Academic, London, 1994.

[5] G. M. Schütz, Solvable Models for Many-Body Systems Far from Equilibrium in C. Domb and J. Lebowitz eds., Phase Transitions and Critical Phenomena, 19: 1251, Academic, London, 2001.

[6] B. Derrida, M. R. Evans, V. Hakim, and V. Pasquir, Exact solution of a 1D exclusion model using a matrix formulation, J. Phys. A., 26: 1493-1517, 1993.

[7] T. Sasamoto, One-dimensional partially asymmetric simple exclusion process with open boundaries: orthogonal polynomials approach, J. Phys. A., 32: 7109-7131, 1999.

[8] M. Uchiyama, T. Sasamoto, and M. Wadati, Asymmetric simple exclusion process with open boundaries and Askey-Wilson polynomials, J. Phys. A., 37: 4985-5002, 2004.

[9] C. Kipnis, Central limit theorem for infinite series of queues and applications to simple exclusion, Ann. Prob., 14: 397-408, 1986.

[10] H. van Beijeren, Fluctuation in the motions of mass and of patterns in one-dimensional hopping model, J. Stat. Phys., 63: 47-58, 1991.

[11] S. N. Majumdar and M. Barma, Tag diffusion in driven systems, growing surfaces, and anomalous fluctuations, Phys. Rev. B, 44: 5306-5308, 1991.

[12] M. Kardar, G. Parisi, and Y. C. Zhang, Dynamic scaling of growing interfaces, Phys. Rev. Lett., 56: 889-892, 1986.

[13] L.-H. Gwa and H. Spohn, Six-vertex model, roughened surfaces, and an asymmetric spin Hamiltonian, Phys. Rev. Lett., 68: 725-728, 1992.

[14] D. Kim, Bethe ansatz solution for crossover scaling functions of the asymmetric XXZ chain and the Kardar-Parisi-Zhang-type growth model, Phys. Rev. E, 52: 3512-3524, 1995.

[15] T. Seppäläinen, A scaling limit for queues in series, Ann. Appl. Prob., 7: 855-872, 1997. 
[16] J. Baik, P. A. Deift, and K. Johansson, On the distribution of the length of the longest increasing subsequence in a random permutation, J. Amer. Math. Soc., 12: 1119-1178, 1999.

[17] K. Johansson, Shape fluctuations and random matrices, Commun. Math. Phys., 209: 437-476, 2000.

[18] J. Baik and E. M. Rains, Algebraic aspects of increasing subsequences, Duke Math. J., 109: 1-65, 2001.

[19] J. Baik and E. M. Rains, The asymptotics of monotone subsequences of involutions, Duke Math. J., 109: 205-281, 2001.

[20] J. Baik and E. M. Rains, Symmetrized random permutations, in P. M. Bleher and A. R. Its, eds., Random Matrix Models and Their Applications, 1-29, Cambridge University Press, 2001.

[21] C. A. Tracy and H. Widom, Level-spacing distributions and the Airy kernel, Commun. Math. Phys., 159: 151-174, 1994.

[22] T. Nagao and T. Sasamoto, Asymmetric simple exclusion process and modified random matrix ensembles, Nucl. Phys. B, 699: 487-502, 2004.

[23] A. Rákos and G. M. Schütz, Current distribution and random matrix ensembles for an integrable asymmetric fragmentation process, J. Stat. Phys., 118: 511-530, 2005.

[24] C. A. Tracy and H. Widom, On orthogonal and symplectic matrix ensembles, Commun. Math. Phys., 177: 727-754, 1996.

[25] M. Prähofer and H. Spohn, Current fluctuations for the totally asymmetric simple exclusion process, In V. Sidoravicius, ed., In and out of equilibrium, Series: Progress in Probability, 51: 185-204, Birkhäuser, 2002.

[26] T. Sasamoto, Spatial correlations of the 1D KPZ surface on a flat substrate, J. Phys. A, 38: L549-L556, 2005.

[27] G. M. Schütz, Exact solution of the master equation for the asymmetric exclusion process, J. Stat. Phys., 88: 427-445, 1997.

[28] T. Seppäläinen, Exact limiting shape for a simplified model of first-passage percolation on the plane, Ann. Prob., 26: 1232-1250, 1998.

[29] K. Johansson, Discrete orthogonal polynomial and the Plancherel measure, Ann. Math., 153: 259-296, 2001.

[30] J. Gravner, C. A. Tracy, and H. Widom, Limit theorems for height fluctuations in a class of discrete space and time growth models, J. Stat. Phys., 102: 1085-1132, 2001. 
[31] J. Gravner, C. A. Tracy, and H. Widom, A growth model in a random environment, Ann. Prob., 30: 1340-1369, 2002.

[32] N. O'Connell, Conditioned random walk and the RSK correspondence, J. Phys. A, 36: 3049-3066, 2003.

[33] N. O'Connell, A path-transformation for random walks and the Robinson-Schensted correspondence, Trans. Am. Math. Soc., 355: 3669-3097, 2003.

[34] A. Borodin and G. Olshanski, Stochastic dynamics related to Plancherel measures on partitions, In V. A. Kaimanovich and A. Lodkin, eds, Representation Theory, Dynamical Systems, and Asymptotic Combinatorics (American Mathematical Society Translations Series 2, Vol. 217), 9-22, 2006.

[35] A. Borodin and G. Olshanski, Markov processes on partitions, Probab. Theory. Relat. Fields, 135: 84-152, 2006.

[36] A. Okounkov and N. Reshetikhin, Correlation function of Schur process with application to local geometry of a random 3-dimensional Young diagram, J. Amer. Math. Soc., 16: 581-603, 2003.

[37] A. Borodin and E. M. Rains, Eynard-Mehta theorem, Schur process, and their Pfaffian analogs, J. Stat. Phys., 121: 291-317, 2006.

[38] K. Johansson, Discrete polynuclear growth and determinantal processes, Commun. Math. Phys., 242: 277-329, 2003.

[39] A. Borodin, P. L. Ferrari, M. Prähofer, Fluctuations in the discrete TASEP with

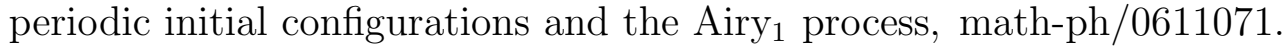

[40] A. M. Povolotsky and V. B. Priezzhev, Determinant solution for the totally asymmetric exclusion process with parallel update, J. Stat. Mech., P07002, 2006.

[41] A. Borodin, P. L. Ferrari, M. Prähofer, and T. Sasamoto, Fluctuation properties of the TASEP with periodic initial configuration, math-ph/0608056.

[42] J. Baik, G. Ben Arous, and S. Péché, Phase transition of the largest eigenvalue for non-null complex sample covariance matrices, Ann. Prob., 33: 1643-1697, 2005.

[43] J. Baik, Painlevé formulas of the limiting distributions for non-null complex sample covariance matrices, Duke Math. J., 133: 205-235, 2006.

[44] A. Rákos and G. M. Schütz, Bethe ansatz and current distribution for the TASEP with particle-dependent hopping rates, Mar. Pro. Relat. Fields, 12: 323-334, 2006.

[45] M. R. Evans, Bose-Einstein condensation in disordered exclusion models and relation to traffic flow, Europhys. Lett., 36: 13-18, 1996. 
[46] M. R. Evans, Exact steady states of disordered hopping particle models with parallel and ordered sequential dynamics, J. Phys. A., 30: 5669-5685, 1997.

[47] T. Seppäläinen and J. Krug, Hydrodynamics and platoon formation for a totally asymmetric exclusion model with particlewise disorder, J. Stat. Phys., 95: 525-567, 1999.

[48] G. E. Andrews, and R. Askey, and R. Roy, Special Functions, Cambridge University Press, 1999.

[49] A. Borodin, and G. Olshanski, Asymmetrics of Plancherel-type random partitions, J. Albebra, 2007, doi:10.1016/j-jalgebra.2006.10.039, math.PR/0610240.

[50] P. J. Forrester, T. Nagao, and G. Honner, Correlations for the orthogonal-unitary and symplectic transitions at the hard and soft edges, Nucl. Phys. B, 553: 601-643, 1999.

[51] A. M. S. Macêdo, Universal parametric correlations at the soft edge of spectrum of random matrix ensembles, Europhys. Lett., 26: 641-646, 1994.

[52] M. Prähofer and H. Spohn, Scale invariance of the PNG droplet and the Airy process, J. Stat. Phys., 108: 1071-1106, 2002.

[53] F. J. Dyson, A Brownian-motion model for the eigenvalues of a random matrix, J. Math. Phys., 3: 1191-1198, 1962.

[54] T. Imamura and T. Sasamoto, Fluctuations of the one-dimensional polynuclear growth model with external sources, Nucl. Phys. B, 699: 503-544, 2004.

[55] T. Imamura and T. Sasamoto, Polynuclear growth model with external source and random matrix model with deterministic source, Phys. Rev. E, 71: 041696, 2005.

[56] J. Baik and E. M. Rains, Limiting distributions for a polynuclear growth model with external sources, J. Stat. Phys., 100: 523-541, 2000.

[57] P. J. Forrester, Painlevé transcendent evaluation of the scaled distribution of the smallest eigenvalue in the Laguerre orthogonal and symplectic ensembles, nlin.SI/0005064.

[58] P. Desrosiers and P. J. Forrester, Asymptotic correlations for Gaussian and Wishart matrices with external source, Int. Math. Res. Not., 2006: 27395, 2006.

[59] B. Eynard and M. L. Mehta, Matrices coupled in a chain: I. Eigenvalue correlations, J. Phys. A, 31: 4449-4456, 1998.

[60] R. P. Stanley, Enumerative Combinatorics Volume 2, Cambridge University Press, 1999.

[61] W. Fulton, Young tableaux, Cambridge University Press, 1999. 
[62] P. L. Ferrari and H. Spohn, Step fluctuations for a faceted crystal, J. Stat. Phys., 113: 1-46, 2003.

[63] K. Johansson, The arctic circle boundary and the Airy process, Ann. Prob., 33: 1-30, 2005. 


\section{Figure Captions}

Fig. 1: Definition of the TASEP with the step initial condition. (a) During each time step, the $i$ th particle can hop to the right with probability $1-q_{i}$. However, $i+2$ th particle cannot hop to the right neighboring site since the site is occupied by $i+1$ th particle. (b) Step initial condition.

Fig. 2: Typical time evolution of the particles from the first to 100th in the TASEP with the step initial condition. The hopping rate of all particles is $0.9(q=0.1)$ except the four defect particles (the first, 25th, 50th and 75th particles) whose hopping rate is 0.8 $(\bar{q}=0.2)$. Fig. (a) illustrates the whole time evolution from $t=0$ through 3000 . Its closeup around $t=200$ and $t=3000$ is shown in (b) and (c) respectively.

Fig. 3: Average position of the tagged particle. The thick line shows (3.11). We divide this line into four regions, where $u$ is near $1 /(1-q), 1 /(1-q)<u<u_{c}, u$ is near $u_{c}$, and $u_{c}<u$. We denote them as region $1,2,3$, and 4 respectively.

Fig. 4: Example of the time evolution of the TASEP and corresponding 01 matrices. In this case, two tables is assigned for one example of the time evolution. Since the second particle can not hop to its right neighboring site between $t=2$ and 3 , there are two possibilities of assigning both 0 and 1 in the $(i, j)=(1,2)$ element of the 01 matrix.

Fig. 5: (a) Example of time evolution of the TASEP and (b) one of its corresponding matrices. Note that a set of many matrices corresponds to (a) and only one of them is shown here.

Fig. 6: (a) Generalized permutation corresponding to Fig. 5(b). We arrange $(i, j)$ where $a(i, j)=1$ following the rule in [60]. (b) The pair $(P, Q)$ obtained from (a) by the dual RSK algorithm.

Fig. 7: Growth of Young diagram obtained from Fig. 5(b) by the dual RSK algorithm. The diagrams $\lambda(i, 4)(1=1, \cdots, 6)$ are obtained from the submatrices $\{a(j, k)\}_{j=1, \cdots, i, k=1, \cdots, 4}$ of Fig. 5(b). The set of the diagrams $\{\lambda(i, 4)\}_{i=1, \cdots, 6}$ corresponds to the $Q$ tableaux in Fig. 6(b). 
Fig. 8: Probability distributions of the scaled position of the 100th particle from the right $(M=100)$ for $t=200$ (a), $t=1000$ (b) and $t=3000$ (c). In these figures, $\times$ represents the data for the case without defect particles and we set the stay rate $q=0.1$. On the other hand, + corresponds to the case where the first particle is a defect particle with $\bar{q}=0.2$ while remaining particles are normal ones with $q=0.1$. The number of samples are 10000 for each case. In (a), both cases belong to the region 2 and are fitted into the GUE Tracy-Widom distribution shown as the dashed line. In (b), the second case belongs to the region 3 since $u=t / M=10=u_{c}$ where $u_{c}=\left(\bar{q}^{2}-2 \bar{q} q+q\right) /(\bar{q}-q)^{2}$ and it is described by the distribution denoted as $\mathrm{GOE}^{2}$ while the first case remains in region 2. In (c), the second cases comes under the region 4 where the distribution is described by Gaussian (dashed line). 
Fig. 1

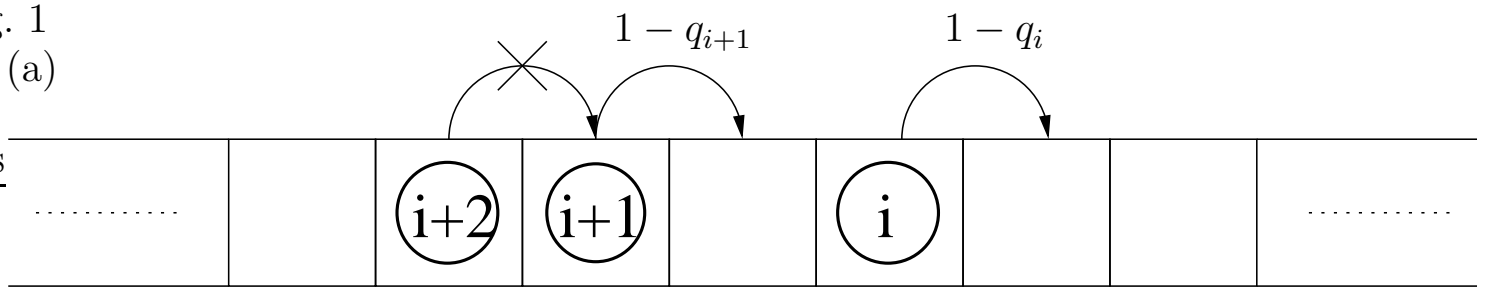

(b)

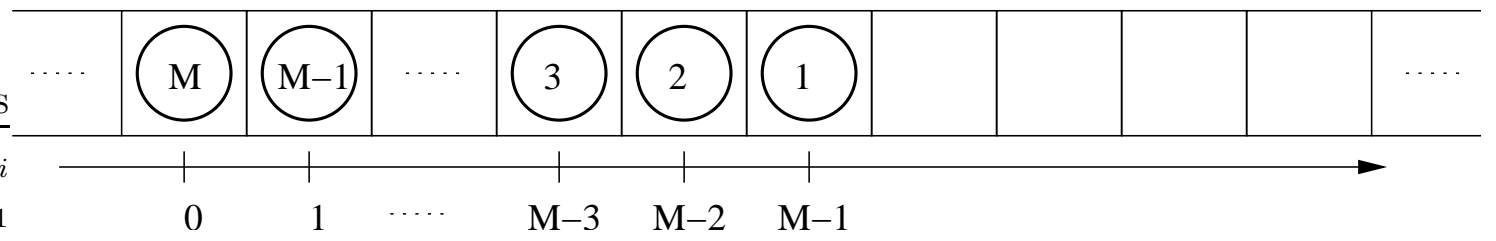

Fig. 2

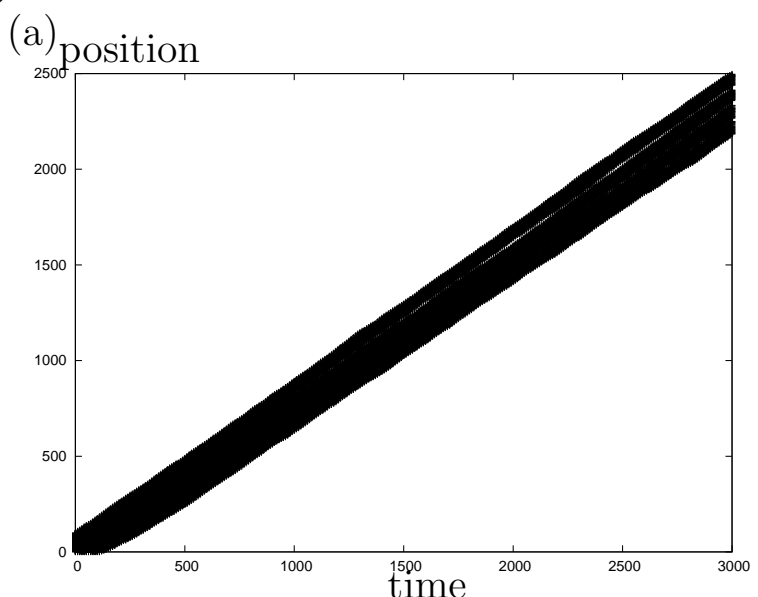

(b) position

(c) position
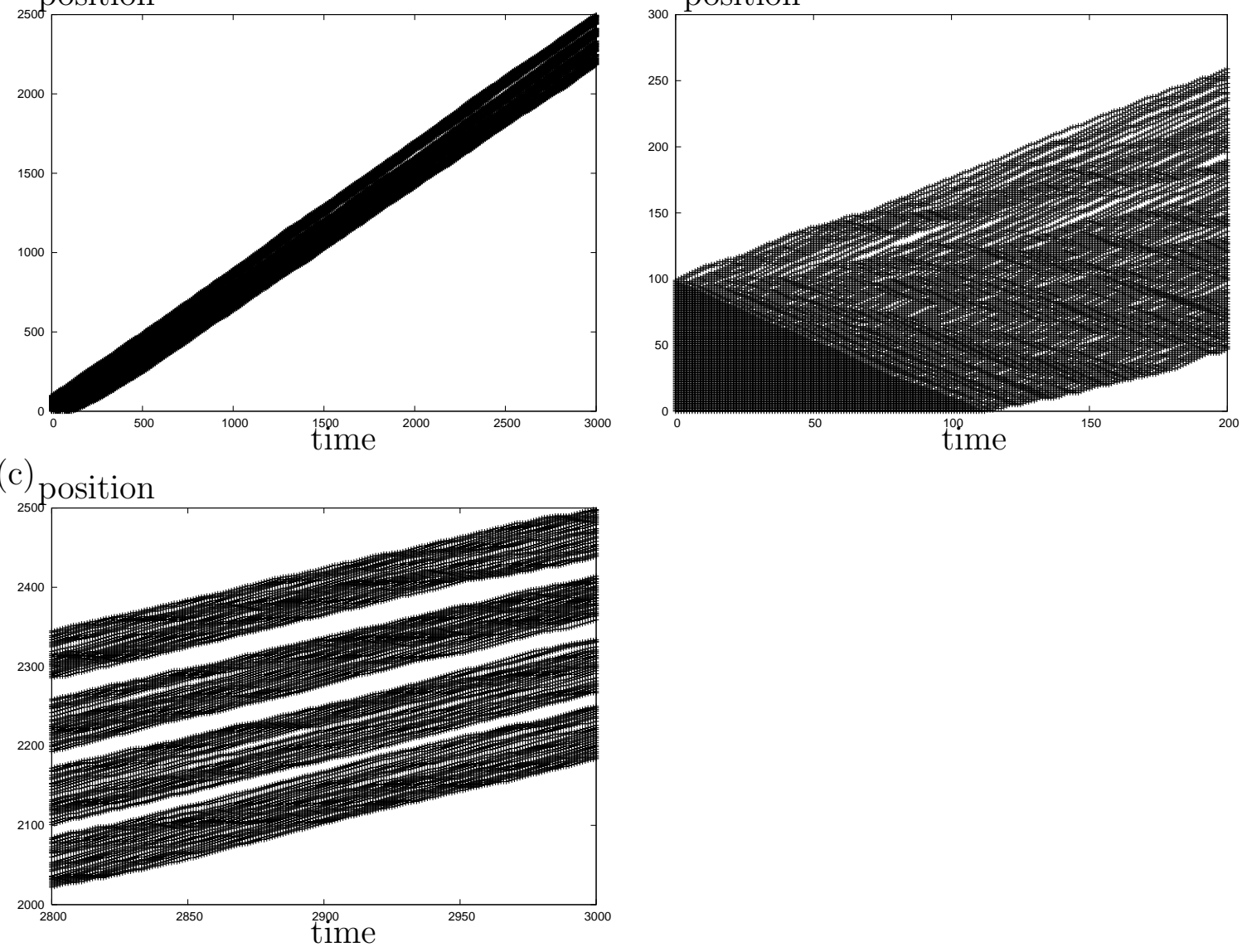
Fig. 3

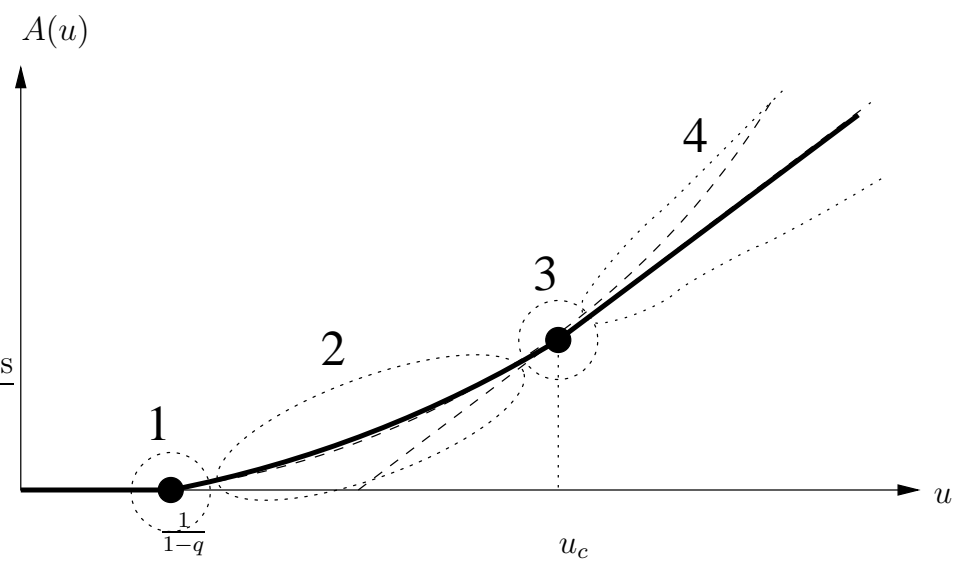

Fig. 4

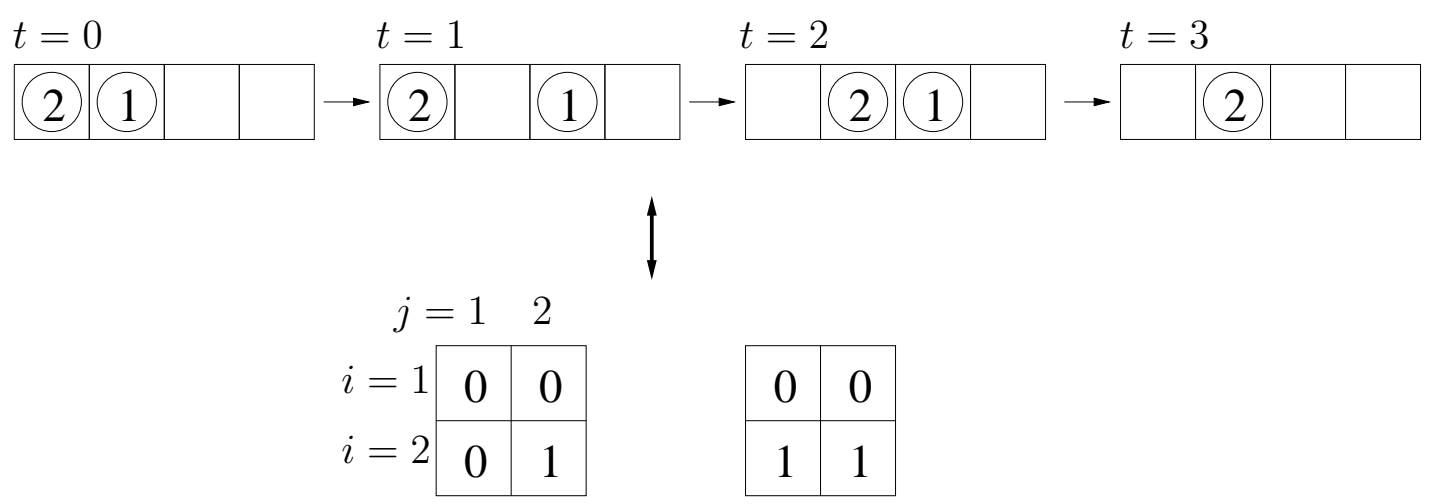

Fig. 5

(a)

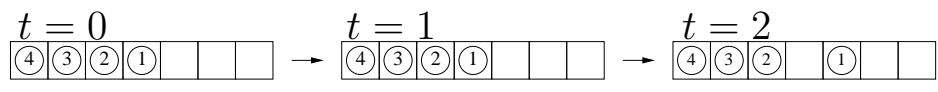

$$
\begin{aligned}
& t=3 \quad t=4 \quad t=5
\end{aligned}
$$

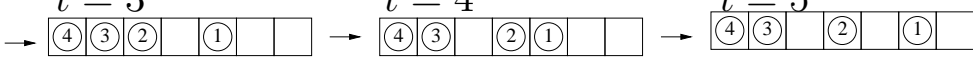

$$
\begin{aligned}
& t=6 \quad t=7 \quad t=8
\end{aligned}
$$

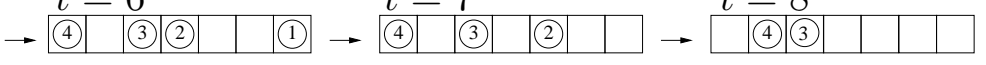

$$
\begin{aligned}
& t=9
\end{aligned}
$$

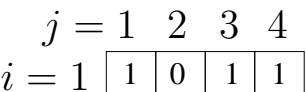

$$
\begin{aligned}
& \begin{array}{l|l|l|l|l|}
i=1 & 1 & 0 & 1 & 1 \\
\hline i=2 & 1 & 1 & 1 & 0 \\
\hline & & 0 & 1 & 0 \\
\hline
\end{array} \\
& i=3 \quad \begin{array}{llllll}
i=3 & 1 & 0 & 1 \\
\hline=4 & 1 & 0 & 0 & 1
\end{array} \\
& i=4 \quad 1 \quad 00|0| 1 \mid \\
& \begin{array}{l|l|l|l|l|}
i=5 & 0 & 0 & 1 & 0 \\
i=6 & 1 & 1 & 0 & 0 \\
\hline
\end{array}
\end{aligned}
$$

(b) 
Fig. 6

(a)

${ }_{j}^{i}\left(\begin{array}{lllllllllllll}1 & 1 & 1 & 2 & 2 & 2 & 3 & 3 & 4 & 4 & 5 & 6 & 6 \\ 1 & 3 & 4 & 1 & 2 & 3 & 2 & 4 & 1 & 4 & 3 & 1 & 2\end{array}\right)$

(b)

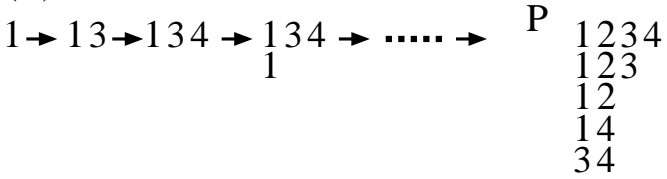

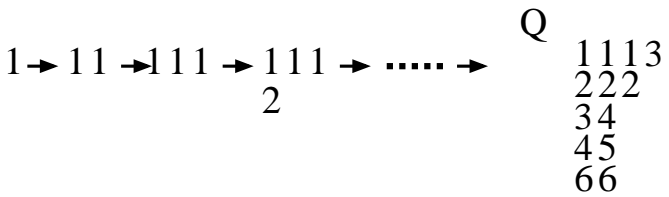

Fig. 7

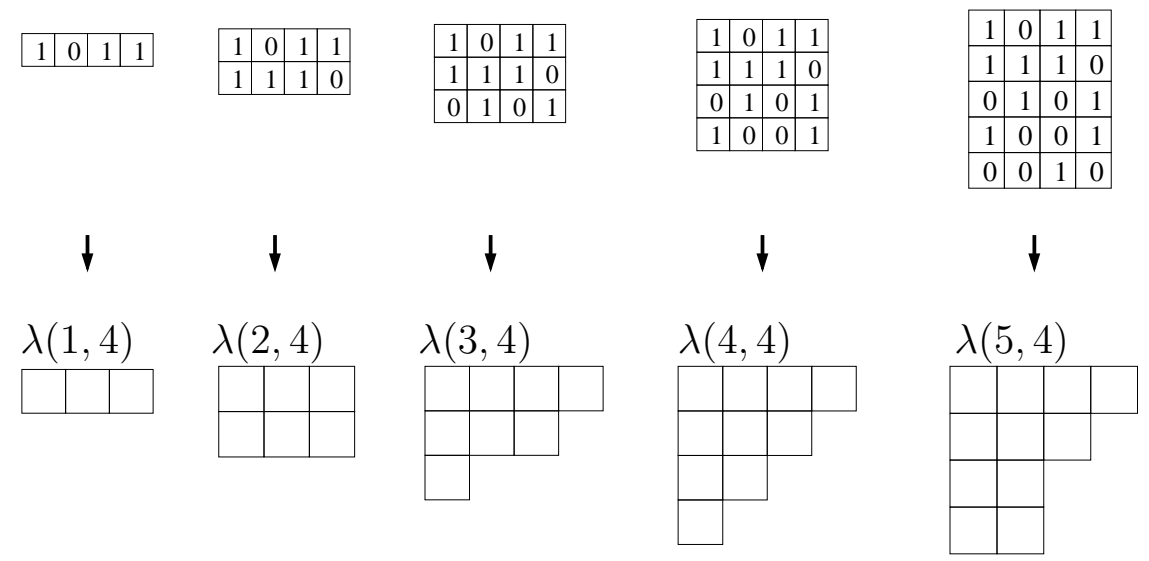

\begin{tabular}{|l|l|l|l|}
\hline 1 & 0 & 1 & 1 \\
\hline 1 & 1 & 1 & 0 \\
\hline 0 & 1 & 0 & 1 \\
\hline 1 & 0 & 0 & 1 \\
\hline 0 & 0 & 1 & 0 \\
\hline 1 & 1 & 0 & 0 \\
\hline \multicolumn{4}{|c}{$\downarrow$} \\
\multicolumn{1}{|c}{} \\
\multicolumn{3}{|c}{}
\end{tabular}

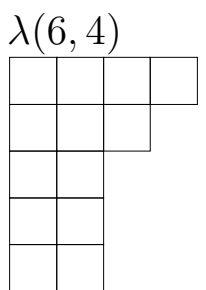

Fig. 8

(a)

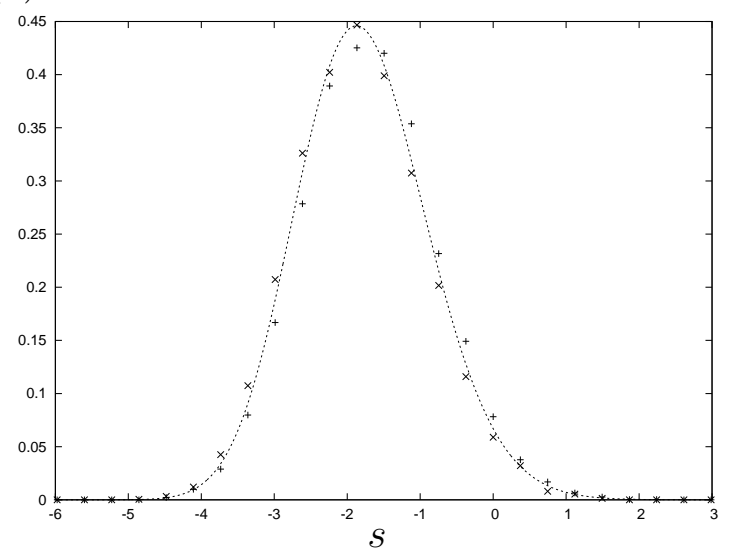

(b)

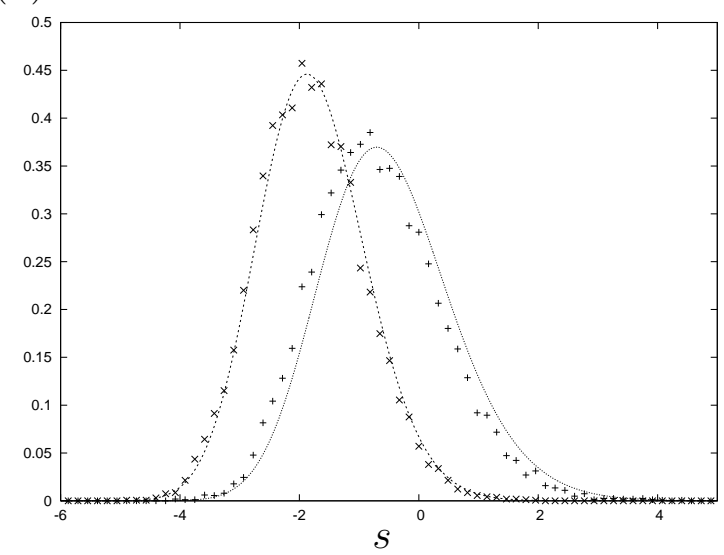


(c)

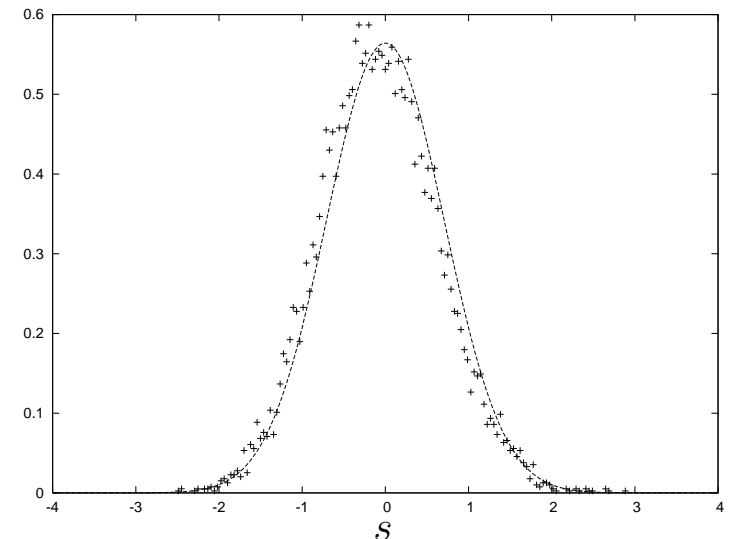

